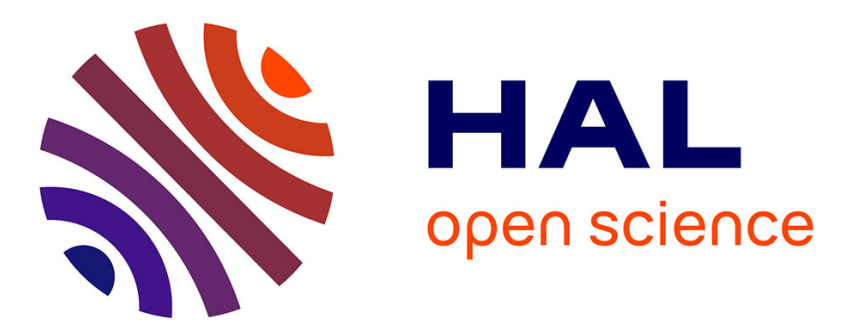

\title{
Global solution and smoothing effect for a non-local regularization of an hyperbolic equation
}

\author{
Jerome Droniou, Thierry Gallouët, Julien Vovelle
}

\section{To cite this version:}

Jerome Droniou, Thierry Gallouët, Julien Vovelle. Global solution and smoothing effect for a nonlocal regularization of an hyperbolic equation. Journal of Evolution Equations, 2003, 3 (3), pp.499-521. 10.1007/s00028-003-0503-1 . hal-00003438

\section{HAL Id: hal-00003438 \\ https://hal.science/hal-00003438}

Submitted on 1 Dec 2004

HAL is a multi-disciplinary open access archive for the deposit and dissemination of scientific research documents, whether they are published or not. The documents may come from teaching and research institutions in France or abroad, or from public or private research centers.
L'archive ouverte pluridisciplinaire HAL, est destinée au dépôt et à la diffusion de documents scientifiques de niveau recherche, publiés ou non, émanant des établissements d'enseignement et de recherche français ou étrangers, des laboratoires publics ou privés. 


\title{
Global solution and smoothing effect for a non-local regularization of an hyperbolic equation
}

\author{
J. Droniou ${ }^{1}$, T. Gallouët ${ }^{2}$, J. Vovelle ${ }^{2}$ \\ $03 / 10 / 2002$
}

\section{Introduction}

We study the problem

$$
\begin{cases}\partial_{t} u(t, x)+\partial_{x}(f(u))(t, x)+g[u(t, \cdot)](x)=0 & t \in] 0, \infty[, x \in \mathbb{R} \\ u(0, x)=u_{0}(x) & x \in \mathbb{R},\end{cases}
$$

where $f \in C^{\infty}(\mathbb{R})$ is such that $f(0)=0$ (there is not loss of generality in assuming this), $u_{0} \in L^{\infty}(\mathbb{R})$ and $g$ is the non-local (in general) operator defined through the Fourier transform by

$$
\left.\left.\mathcal{F}(g[u(t, \cdot)])(\xi)=|\xi|^{\lambda} \mathcal{F}(u(t, \cdot))(\xi), \quad \text { with } \lambda \in\right] 1,2\right] .
$$

Remark 1.1 We could also very well study a multi-dimensional scalar equation, that is to say on $\mathbb{R}^{N}$ instead of $\mathbb{R}$. All the methods and results presented below would apply; but this would lead to more technical manipulations so, for the sake of clarity, we have chosen to fully describe only the mono-dimensional case.

The interest of such an equation (namely Equation (1.1)) was pointed out to us by Paul Clavin in the context of pattern formation in detonation waves. The study of detonations leads, in a first approximation, to nonlinear hyperbolic equations. As it is well known, the solutions of such equations may develop discontinuities in finite time. A theory of existence and uniqueness of (entropy weak) solutions to Equation (1.1) with $g=0$, in the $L^{\infty}$ framework, is known since the work of Krushkov ([Kru70], see also [Vol67]). The case of a parabolic regularization (of a nonlinear hyperbolic equation) is often considered and used to prove the Krushkov result; it corresponds to (1.1) with $\lambda=2$. In this case, existence and uniqueness of a solution is also well known along with a regularizing effect. However, it appears that the choice of $\lambda=2$ is not quite natural, at least for the problem of detonation (see [CD02], [CH01], [CD01]) where it seems more natural to consider a nonlocal term as $g[u]$ with $\lambda$ close to 1 but greater than 1 (although the case $\lambda=1$ is also of

\footnotetext{
${ }^{1}$ Département de Mathématiques, CC 051, Université Montpellier II, Place Eugène Bataillon, 34095 Montpellier cedex 5, France. email: droniou@math.univ-montp2.fr

${ }^{2}$ CMI, Université de Provence, 39 rue Joliot-Curie, 13453 Marseille cedex 13, France. email: gallouet@cmi.univ-mrs.fr, vovelle@cmi.univ-mrs.fr
} 
interest but more complicated). This term corresponds to some spatial fractional derivative of $u$ of order $\lambda$. The main motivation of this paper is therefore to prove existence and uniqueness of the solution to (1.1) in the $L^{\infty}$ framework as well as a regularizing effect (a regularizing effect which is well-known in the case $\lambda=2$, as it is said above). In particular, the solution will be $C^{\infty}$ in space and time for $t>0$. We also prove the so called "maximum principle", namely the fact that the solution takes values between the maximum and the minimum values of the initial data, and a property of " $L^{1}$ contraction" on the solutions, which is the fact that, for any time, the $L^{1}$ norm of the difference of two solutions with different initial data is bounded by the $L^{1}$ norm (if it exists) of the difference of the initial data.

A major difficulty is due to the nonlocal character of $g[u]$ if $\lambda \in] 1,2[$; this prevents the classical way to prove the maximum principle, which leads to an $L^{\infty}$ a priori bound on the solution (a crucial estimate to obtain global solutions). It is interesting to notice that the hypothesis $\lambda \leq 2$ is necessary for the maximum principle. Indeed, the maximum principle is no longer true in general for $\lambda>2$. However, the regularizing effect is still true for $\lambda>2$, a property which is probably not verified if $\lambda<1$. The case $\lambda=1$ is not so clear and needs an additional work. Indeed, for the study of detonation waves, our result has to be viewed as a preliminary result or, at least, as a study of a very simplified case. Realistic models are much more complicated. In particular, it seems that $\lambda$ is actually depending on the unknown and, even if $\lambda>1, \lambda$ is probably not bounded from below by some $\lambda_{0}>1$. The possibility to generalize our result to such a case is not manifest.

We first prove (Section 4) the uniqueness of a "weak" solution (solution in the sense of Definition 3.1 below). Then, assuming the existence of a "weak" solution, we prove (Section 5) the regularizing effect (the equation is then satisfied in a classical sense). The results of these two sections are in fact true for any $\lambda>1$. In Section 6, the existence result is given, using a splitting method. The use of splitting methods is classical, in particular to define numerical schemes, but is not usual to prove an existence result as it is done here. In this section, the central argument is the proof of the maximum principle (which is limited to $\lambda \leq 2$ ).

Here is our main result.

Theorem 1.1 If $u_{0} \in L^{\infty}(\mathbb{R})$, then there exists a unique solution $u$ to (1.1) on ] $0, \infty[$ (in the sense of Definition 3.1, see below). Moreover, this solution satisfies:

i) $u \in C^{\infty}(] 0, \infty[\times \mathbb{R})$ and all its derivatives are bounded on $] t_{0}, \infty[\times \mathbb{R}$ for all $t_{0}>0$,

ii) for all $t>0,\|u(t)\|_{L^{\infty}(\mathbb{R})} \leq\left\|u_{0}\right\|_{L^{\infty}(\mathbb{R})}$ and, in fact, $u$ takes its values between the essential lower and upper bounds of $u_{0}$,

iii) $u$ satisfies $\partial_{t} u+\partial_{x}(f(u))+g[u]=0$ in the classical sense $(g[u]$ being properly defined by Proposition 5.2). 


$$
\text { iv) } u(t) \rightarrow u_{0} \text {, as } t \rightarrow 0 \text {, in } L^{\infty}(\mathbb{R}) \text { weak-* and in } L_{\mathrm{loc}}^{p}(\mathbb{R}) \text { for all } p \in[1, \infty[\text {. }
$$

Remark 1.2 In the course of our study of (1.1), we will also see that, if $u_{0} \in$ $L^{\infty}(\mathbb{R}) \cap L^{1}(\mathbb{R})$, then the solution $u$ to (1.1) satisfies, for all $t>0$ : $\|u(t)\|_{L^{1}(\mathbb{R})} \leq$ $\left\|u_{0}\right\|_{L^{1}(\mathbb{R})}$.

We will also see that (1.1) has a $L^{1}$ contraction property: if $\left(u_{0}, v_{0}\right) \in L^{\infty}(\mathbb{R})$ are such that $u_{0}-v_{0} \in L^{1}(\mathbb{R})$, then, denoting by $u$ and $v$ the solutions to (1.1) corresponding to initial conditions $u_{0}$ and $v_{0}$, we have, for all $t>0: \| u(t)-$ $v(t)\left\|_{L^{1}(\mathbb{R})} \leq\right\| u_{0}-v_{0} \|_{L^{1}(\mathbb{R})}$.

\section{Properties of the kernel of $g$}

Using the Fourier transform, we see that the semi-group generated by $g$ is formally given by the convolution with the kernel (defined for $t>0$ and $x \in \mathbb{R}$ )

$$
K(t, x)=\mathcal{F}^{-1}\left(e^{-t|\cdot|^{\lambda}}\right)(x)=\int_{\mathbb{R}} e^{2 i \pi x \xi} e^{-t|\xi|^{\lambda}} d \xi=\mathcal{F}\left(e^{-t|\cdot|^{\lambda}}\right)(x) .
$$

The function $\xi \in \mathbb{R} \rightarrow e^{-t|\xi|^{\lambda}}$ being real-valued and even, $K$ is real-valued (in the sequel, we consider only real-valued solutions to (1.1)).

The most important property of $K$ is its nonnegativity. For the sake of completeness, we give here a sketch of the proof of this result, but notice that it is a well-known result since a rather long time now. We refer to the work of Lévy for example [Lév25]. Also notice that we study the question of the non-negativity of the kernel $K$ because it is the issue at stake in the analysis of a maximum principle for the equation $u_{t}+g[u]=0$. From this point of view, we shall make reference to the work of Courrège and coworkers (see [BCP68] and references therein) who give a characterization of a large class of pseudo-differential operators satisfying the positive maximum principle and also, more recently, to the work of Farkas, Jacob, Schilling [FJS01] (see also Hoh [Hoh95]).

Lemma 2.1 If $\lambda \in] 0,2]$ then, for all $(t, x) \in] 0, \infty[\times \mathbb{R}$, we have $K(t, x) \geq 0$.

\section{Proof of Lemma 2.1}

If $\lambda=2$, it is well-known that $K(t, x)=(\pi / t)^{1 / 2} e^{-\frac{\pi^{2}}{t} \xi^{2}}$, which implies the result. Assume now that $\lambda \in] 0,2\left[\right.$ and let $f(x)=A|x|^{-1-\lambda} \mathbf{1}_{\mathbb{R} \backslash]-1,1[}(x)$, with $A>0$ such that $\int_{\mathbb{R}} f(x) d x=1$. Since $f$ is even with integral equal to 1 , we have

$$
\mathcal{F}(f)(\xi)=1+\int_{\mathbb{R}}(\cos (2 \pi x \xi)-1) f(x) d x=1+A|\xi|^{\lambda} \int_{|y| \geq|\xi|} \frac{\cos (2 \pi y)-1}{|y|^{1+\lambda}} d y .
$$

Since $\cos (2 \pi y)-1=\mathcal{O}\left(|y|^{2}\right)$ on the neighborhood of 0 and $\lambda<2$, the dominated convergence theorem gives

$$
\int_{|y| \geq|\xi|} \frac{\cos (2 \pi y)-1}{|y|^{1+\lambda}} d y \rightarrow I:=\int_{\mathbb{R}} \frac{\cos (2 \pi y)-1}{|y|^{1+\lambda}} d y<0 \quad \text { as } \xi \rightarrow 0 .
$$


Hence, $\mathcal{F}(f)(\xi)=1-c|\xi|^{\lambda}(1+\omega(\xi))$ with $c=-A I>0$ and $\lim _{\xi \rightarrow 0} \omega(\xi)=0$.

Define $f_{n}(x)=n^{1 / \lambda} f * f * \cdots * f\left(n^{1 / \lambda} x\right)$, the convolution product being taken $n$ times. By the properties of the Fourier transform with respect to the convolution product, we have, for all $\xi \in \mathbb{R}$,

$$
\mathcal{F}\left(f_{n}\right)(\xi)=\left(\mathcal{F}(f)\left(n^{-1 / \lambda} \xi\right)\right)^{n}=\left(1-c n^{-1}|\xi|^{\lambda}\left(1+\omega\left(n^{-1 / \lambda} \xi\right)\right)\right)^{n} \rightarrow e^{-c|\xi|^{\lambda}}
$$

as $n \rightarrow \infty$. Since $\left(\mathcal{F}\left(f_{n}\right)\right)_{n \geq 1}$ is bounded by 1 (the $L^{1}$-norm of $f_{n}$ for all $n \geq 1$ ), this convergence is also true in $\mathcal{S}^{\prime}(\mathbb{R})$ and, taking the inverse Fourier transform, we see that $f_{n} \rightarrow \mathcal{F}^{-1}\left(e^{-c|\cdot|^{\lambda}}\right)=K(c, \cdot)$ in $\mathcal{S}^{\prime}(\mathbb{R})$ as $n \rightarrow \infty$. $f_{n}$ being nonnegative for all $n$, we deduce that $K$ is nonnegative on $\{c\} \times \mathbb{R}$; the homogeneity property (2.1) below concludes then the proof of the lemma.

Here are some other important properties of $K$ :

$$
\forall(t, x) \in] 0, \infty\left[\times \mathbb{R}, \quad K(t, x)=\frac{1}{t^{1 / \lambda}} K\left(1, \frac{x}{t^{1 / \lambda}}\right) .\right.
$$

$K$ is $C^{\infty}$ on $] 0, \infty\left[\times \mathbb{R}\right.$ and, for all $m \geq 0$, there exists $B_{m}$ such that

$$
\forall(t, x) \in] 0, \infty\left[\times \mathbb{R}, \quad\left|\partial_{x}^{m} K(t, x)\right| \leq \frac{1}{t^{(1+m) / \lambda}} \frac{B_{m}}{\left(1+t^{-2 / \lambda}|x|^{2}\right)} .\right.
$$

$(K(t, \cdot))_{t>0}$ is, as $t \rightarrow 0$, an approximate unit (in particular, $\|K(t, \cdot)\|_{L^{1}(\mathbb{R})}=1$ for all $t>0$ ).

$$
\exists \mathcal{K}_{1} \text { such that, for all } t>0, \quad\left\|\partial_{x} K(t, \cdot)\right\|_{L^{1}(\mathbb{R})}=\mathcal{K}_{1} t^{-1 / \lambda} \text {. }
$$

$$
\begin{gathered}
\forall(a, b) \in] 0, \infty[, K(a, \cdot) * K(b, \cdot)=K(a+b, \cdot) \\
\text { and } K(a, \cdot) * \partial_{x} K(b, \cdot)=\partial_{x} K(a+b, \cdot) .
\end{gathered}
$$

\section{Proof of these properties}

Equation (2.1) is obtained thanks to the change of variable $\xi=t^{-1 / \lambda} \eta$ in the integral defining $K$.

The regularity of $K$ is an immediate application of the theorem of derivation under the integral sign. To prove the second part of (2.2), we write $\partial_{x}^{m} K(1, x)=$ $\int_{\mathbb{R}}(2 i \pi \xi)^{m} e^{-|\xi|^{\lambda}} e^{2 i \pi x \xi} d \xi$; since $\lambda>1$, the first two derivatives of $\xi \rightarrow \xi^{m} e^{-|\xi|^{\lambda}}$ are integrable on $\mathbb{R}$ and we can make two integrations by parts to obtain $\partial_{x}^{m} K(1, x)=$ $\mathcal{O}\left(1 / x^{2}\right)$ on $\mathbb{R} ; \partial_{x}^{m} K(1, \cdot)$ being bounded on $\mathbb{R}$, we deduce the estimate of $(2.2)$ for $t=1$; the general case $t>0$ comes from the case $t=1$ and (2.1).

Since $K(1, \cdot) \geq 0$, we have $\|K(1, \cdot)\|_{L^{1}(\mathbb{R})}=\int_{\mathbb{R}} K(1, x) d x=\mathcal{F}(K(1, \cdot))(0)=$ $e^{-|0|^{\lambda}}=1$ and (2.3) is thus a consequence of (2.1).

The estimate (2.4) comes from the derivation of (2.1) and from the change of variable $y=t^{-1 / \lambda} x$ in the computation of $\left\|\partial_{x} K\left(1, \cdot / t^{1 / \lambda}\right)\right\|_{L^{1}(\mathbb{R})}$.

The identity (2.5), which translates the fact that the convolution with $K(t)$ is the semi-group generated by $g$, can be directly checked via Fourier transform. 
Let us also give some continuity results related to $K$.

Lemma $2.2 \quad$ i) If $u_{0} \in L^{1}(\mathbb{R})$, then $t \in\left[0, \infty\left[\rightarrow K(t, \cdot) * u_{0}\right.\right.$ is continuous $\left[0, \infty\left[\rightarrow L^{1}(\mathbb{R})\right.\right.$ (with value $u_{0}$ at $\left.t=0\right)$.

ii) Let $T>0$ and $\left.\left(t_{0}, x_{0}\right) \in\right] 0, T\left[\times \mathbb{R}\right.$. If $v \in C_{b}(] 0, T[\times \mathbb{R})$, then

a) for all $s_{0}>0, K(s, \cdot) * v(t, \cdot)(x) \rightarrow K\left(s_{0}, \cdot\right) * v\left(t_{0}, \cdot\right)\left(x_{0}\right)$ as $s \rightarrow s_{0}$, $t \rightarrow t_{0}$ and $x \rightarrow x_{0}$,

b) $K(s, \cdot) * v(t, \cdot)(x) \rightarrow v\left(t_{0}, x_{0}\right)$ as $s \rightarrow 0, t \rightarrow t_{0}$ and $x \rightarrow x_{0}$.

All these properties are either classical results of approximate units or consequences of the estimate in (2.2) (with $m=0$ ) and of the dominated convergence theorem. We do not give a precise proof of these results.

\section{Definition and first properties of the solutions}

The idea, to study (1.1), is to search for a solution to $\partial_{t} u+g[u]=-\partial_{x}(f(u))$ using Duhamel's formula: a solution to this equation is formally given by $u(t, x)=$ $K(t) * u_{0}(x)-\int_{0}^{t} K(t-s) * \partial_{x}(f(u(s, \cdot)))(x) d s$. By putting the derivative of $f(u)$ on $K$, we are led to the following definition.

Definition 3.1 Let $u_{0} \in L^{\infty}(\mathbb{R})$ and $T>0$ or $T=\infty$. A solution to (1.1) on ] $0, T\left[\right.$ is a function $u \in L^{\infty}(] 0, T[\times \mathbb{R})$ which satisfies, for a.e. $\left.(t, x) \in\right] 0, T[\times \mathbb{R}$,

$$
u(t, x)=K(t, \cdot) * u_{0}(x)-\int_{0}^{t} \partial_{x} K(t-s, \cdot) * f(u(s, \cdot))(x) d s .
$$

The following proposition shows that all the terms in (3.1) are well-defined.

Proposition 3.1 Let $u_{0} \in L^{\infty}(\mathbb{R})$ and $T>0$. If $v \in L^{\infty}(] 0, T[\times \mathbb{R})$, then

$$
u:(t, x) \in] 0, T\left[\times \mathbb{R} \rightarrow K(t, \cdot) * u_{0}(x)+\int_{0}^{t} \partial_{x} K(t-s, \cdot) * v(s, \cdot) d s\right.
$$

defines a function in $C_{b}(] 0, T[\times \mathbb{R})$ and we have, for all $\left.t_{0} \in\right] 0, T[$, all $x \in \mathbb{R}$ and all $t \in] 0, T-t_{0}[$,

$$
u\left(t_{0}+t, x\right)=K(t, \cdot) * u\left(t_{0}, \cdot\right)(x)+\int_{0}^{t} \partial_{x} K(t-s, \cdot) * v\left(t_{0}+s, \cdot\right)(x) d s .
$$




\section{Proof of Proposition 3.1}

Step 1: first term of $u$.

Since $u_{0} \in L^{\infty}(\mathbb{R})$ and, for $t>0, K(t, \cdot) \in L^{1}(\mathbb{R}), K(t, \cdot) * u_{0}$ is well-defined and, by Young's inequalities for the convolution and $(2.3)$, we have

$$
\forall(t, x) \in] 0, \infty\left[\times \mathbb{R},\left|K(t, \cdot) * u_{0}(x)\right| \leq\left\|u_{0}\right\|_{L^{\infty}(\mathbb{R})} .\right.
$$

Let $\left.t_{0} \in\right] 0, T\left[\right.$ and $x_{0} \in \mathbb{R}$; for all $0<t_{0}<T<\infty$, by $(2.2)$, we can write

$$
\forall(t, x, y) \in] t_{0}, T\left[\times \mathbb{R} \times \mathbb{R}, \quad|K(t, x-y)| \leq \frac{C_{1}}{C_{2}+|x-y|^{2}}\right.
$$

where $C_{1}>0$ and $C_{2}>0$ only depend on $\left(t_{0}, T\right)$. We have $\left|y-x_{0}\right|^{2} \leq 2 \mid y-$ $\left.x\right|^{2}+2\left|x_{0}-x\right|^{2}$, so that $|y-x|^{2} \geq \frac{1}{2}\left|y-x_{0}\right|^{2}-\left|x_{0}-x\right|^{2}$. For all $x \in \mathbb{R}$ such that $\left|x-x_{0}\right|^{2} \leq C_{2} / 2$, for all $\left.t \in\right] t_{0}, T[$ and all $y \in \mathbb{R},(3.4)$ gives

$$
|K(t, x-y)| \leq \frac{C_{1}}{C_{2}+\frac{1}{2}\left|x_{0}-y\right|^{2}-\left|x_{0}-x\right|^{2}} \leq \frac{C_{1}}{\left(C_{2} / 2\right)+\frac{1}{2}\left|x_{0}-y\right|^{2}}=F(y)
$$

with $F \in L^{1}(\mathbb{R})$. Since $u_{0}$ is bounded and $K$ is continuous, the theorem of continuity under the integral sign gives the continuity of $(t, x) \rightarrow K(t, \cdot) * u_{0}(x)$.

Step 2: the second term of $u$.

Define $G: \mathbb{R} \times \mathbb{R} \rightarrow \mathbb{R}$ and $H: \mathbb{R} \times \mathbb{R} \rightarrow \mathbb{R}$ by: for all $x \in \mathbb{R}$,

$$
\begin{aligned}
& G(t, x)=\partial_{x} K(t, x) \mathbf{1}_{] 0, T}(t) \text { if } t>0, \quad G(t, x)=0 \text { if } t \leq 0, \\
& H(t, x)=v(t, x) \text { if } t \in] 0, T[, \quad H(t, x)=0 \text { if } t \in \mathbb{R} \backslash] 0, T[.
\end{aligned}
$$

We notice that $G \in L^{1}(\mathbb{R} \times \mathbb{R})$; indeed, by Fubini-Tonelli's theorem and (2.4),

$$
\int_{\mathbb{R} \times \mathbb{R}}|G(t, x)| d x d t \leq \mathcal{K}_{1} \int_{0}^{T} t^{-1 / \lambda} d t=\frac{\lambda \mathcal{K}_{1}}{\lambda-1} T^{1-\frac{1}{\lambda}}<\infty .
$$

The function $H$ is clearly in $L^{\infty}(\mathbb{R} \times \mathbb{R})$, being bounded by $\|v\|_{L^{\infty}(10, T[\times \mathbb{R})}$. Thus, denoting by $\star$ the convolution in $\mathbb{R} \times \mathbb{R}, G \star H$ is well-defined, bounded and uniformly continuous on $\mathbb{R} \times \mathbb{R}$; moreover, by (3.5),

$$
\|G \star H\|_{C_{b}(\mathbb{R} \times \mathbb{R})} \leq\|G\|_{L^{1}(\mathbb{R} \times \mathbb{R})}\|H\|_{L^{\infty}(\mathbb{R} \times \mathbb{R})} \leq \frac{\lambda \mathcal{K}_{1}}{\lambda-1} T^{1-\frac{1}{\lambda}}\|v\|_{L^{\infty}(] 0, T[\times \mathbb{R})} .
$$

By Fubini's theorem, one checks that, for all $t \in] 0, T[$ and all $x \in \mathbb{R}, G \star H(t, x)=$ $\int_{0}^{t} \partial_{x} K(t-s, \cdot) * v(s, \cdot)(x) d s$, and the second term of $u$ is thus continuous and bounded on $] 0, T[\times \mathbb{R}$.

We notice that, thanks to (3.3) and (3.6),

$$
\|u\|_{C_{b}(] 0, T[\times \mathbb{R})} \leq\left\|u_{0}\right\|_{L^{\infty}(\mathbb{R})}+\frac{\lambda \mathcal{K}_{1}}{\lambda-1} T^{1-\frac{1}{\lambda}}\|v\|_{L^{\infty}(] 0, T[\times \mathbb{R})} .
$$


Step 3: To prove (3.2), we make the change of variable $\tau=t_{0}+s$ in the last term of this equation, we use Fubini's theorem (thanks to (2.4)) to permute the convolution by $K(t, \cdot)$ and the integral sign in $u\left(t_{0}, \cdot\right)$ and we apply $(2.5)$.

As an immediate consequence of this proposition, we have:

Corollary 3.1 Let $u_{0} \in L^{\infty}(\mathbb{R})$ and $T>0$ or $T=\infty$. If $u$ is a solution to (1.1) on $] 0, T\left[\right.$, then $u \in C_{b}(] 0, T[\times \mathbb{R})$ and $u$ satisfies (3.1) for all $\left.(t, x) \in\right] 0, T[\times \mathbb{R}$. Moreover, for all $\left.t_{0} \in\right] 0, T[$ and all $(t, x) \in] 0, T-t_{0}[\times \mathbb{R}$,

$$
u\left(t_{0}+t, x\right)=K(t, \cdot) * u\left(t_{0}, \cdot\right)(x)-\int_{0}^{t} \partial_{x} K(t-s, \cdot) * f\left(u\left(t_{0}+s, \cdot\right)\right)(x) d s,
$$

i.e. $u\left(t_{0}+\cdot, \cdot\right)$ is a solution to (1.1) on $] 0, T-t_{0}\left[\right.$ with $u\left(t_{0}, \cdot\right)$ instead of $u_{0}$.

To conclude this study of the first properties of the solutions, we prove item iv) of Theorem 1.1.

Proof of item iv) in Theorem 1.1

Suppose that $u$ is a solution to (1.1) on ]0,T[. Since $f(u)$ is bounded, we have, for all $(t, x) \in] 0, T[\times \mathbb{R}$, by $(2.4)$,

$$
\left|\int_{0}^{t} \partial_{x} K(t-s, \cdot) * f(u(s, \cdot))(x) d s\right| \leq \mathcal{K}_{1}\|f(u)\|_{\infty} \int_{0}^{t} \frac{1}{(t-s)^{1 / \lambda}} d s=C t^{1-\frac{1}{\lambda}}
$$

where $C$ does not depend on $t$; hence, the last term of $(3.1)$ tends to 0 in $L^{\infty}(\mathbb{R})$ as $t \rightarrow 0$. By classical properties of the approximate units, the first term in the right-hand side of (3.1) converges as wanted to $u_{0}$ and the proof is complete.

\section{Uniqueness of the solution}

Theorem 4.1 Let $u_{0} \in L^{\infty}(\mathbb{R})$ and $T>0$ or $T=\infty$. There exists at most one solution to (1.1) on ]0,T[ in the sense of Definition 3.1.

\section{Proof of Theorem 4.1}

Step 1: we first prove a local uniqueness result. Denote by $\operatorname{Lip}_{R}(f)$ a lipschitz constant of $f$ on $[-R, R]$. Let $T_{1}>0$. For all $u$ and $v$ solutions to (1.1) on $] 0, T_{1}[$ bounded by $R$, by (3.1) and (2.4), we have

$$
|u(t, x)-v(t, x)| \leq \frac{\lambda \mathcal{K}_{1}}{\lambda-1} T_{1}^{1-\frac{1}{\lambda}} \operatorname{Lip}_{R}(f)\|u-v\|_{\infty}=k\left(T_{1}, R\right)\|u-v\|_{\infty} .
$$

There exists $T_{0}>0$ only depending on $R$ such that, if $T_{1} \leq T_{0}$, we have $k\left(T_{1}, R\right)<$ 1 ; for $T_{1} \leq T_{0}$, there exists therefore at most one solution to (1.1) on ]0, $T_{1}$ [ bounded by $R$. 
Step 2: proof of the uniqueness result.

Let $u$ and $v$ be two solutions to (1.1) on $] 0, T\left[\right.$. Take $R=\max \left(\|u\|_{\infty},\|v\|_{\infty}\right)$; let $T_{0}$ be given by Step 1 for $R$. By Step 1 , since $u$ and $v$ are bounded by $R, u=v$ on $] 0, \inf \left(T, T_{0}\right)[\times \mathbb{R}$.

Let $T^{\prime}=\sup \{t \in] 0, T[\mid u=v$ on $] 0, t[\times \mathbb{R}\} \geq \inf \left(T, T_{0}\right)$, and suppose that $T^{\prime}<T$. By definition of $T^{\prime}$, and since $u$ and $v$ are continuous on $] 0, T[\times \mathbb{R}$, we have $u\left(T^{\prime}, \cdot\right)=v\left(T^{\prime}, \cdot\right)$ on $\mathbb{R}$. By Corollary 3.1, $u\left(T^{\prime}+\cdot, \cdot\right)$ and $v\left(T^{\prime}+\cdot, \cdot\right)$ are two solutions to $(1.1)$ on $] 0, T-T^{\prime}$ [ with the same initial condition $u\left(T^{\prime}, \cdot\right)=v\left(T^{\prime}, \cdot\right)$. These solutions being bounded by $R$, Step 1 shows that $u\left(T^{\prime}+\cdot, \cdot\right)=v\left(T^{\prime}+\cdot, \cdot\right)$ on $] 0, \inf \left(T_{0}, T-T^{\prime}\right)\left[\times \mathbb{R}\right.$, which is a contradiction with the definition of $T^{\prime}$.

\section{$5 \quad$ Regularizing effect}

\subsection{Spatial regularity}

If we formally differentiate (3.1) with respect to $x$, we see that the spatial derivatives of $u$ satisfy integral equations; the following theorem gives some properties on these integral equations.

Proposition 5.1 Let $M>0$ and $F:(t, x, \zeta) \in] 0, M[\times \mathbb{R} \times \mathbb{R} \rightarrow F(t, x, \zeta) \in \mathbb{R}$ be continuous; we suppose that $\partial_{x} F, \partial_{\zeta} F, \partial_{\zeta} \partial_{x} F$ and $\partial_{\zeta} \partial_{\zeta} F$ exist and are continuous on $] 0, M[\times \mathbb{R} \times \mathbb{R} ;$ we also suppose that there exists $\omega:] 0, \infty\left[\rightarrow \mathbb{R}^{+}\right.$such that, for all $L>0, F$ and these derivatives are bounded on $] 0, M[\times \mathbb{R} \times[-L, L]$ by $\omega(L)$.

Let $R_{0}>0$ and $R=\left(2+\mathcal{K}_{1}\right) R_{0}$. Then there exists $T_{0}>0$ only depending on $\left(R_{0}, \omega\right)$ such that, if $T=\inf \left(M, T_{0}\right)$ and $v_{0} \in L^{\infty}(\mathbb{R})$ satisfies $\left\|v_{0}\right\|_{L^{\infty}(\mathbb{R})} \leq R_{0}$, there exists a unique $v \in C_{b}(] 0, T[\times \mathbb{R})$ bounded by $R$ and such that

$$
v(t, x)=K(t, \cdot) * v_{0}(x)+\int_{0}^{t} \partial_{x} K(t-s, \cdot) * F(s, \cdot, v(s, \cdot))(x) d s
$$

Moreover, $\partial_{x} v \in C(] 0, T[\times \mathbb{R})$ and, for all $\left.a \in\right] 0, T\left[,\left\|\partial_{x} v\right\|_{C_{b}(] a, T[\times \mathbb{R})} \leq R a^{-1 / \lambda}\right.$.

\section{Proof of Proposition 5.1}

The idea is to use a fixed point theorem. Let, for $T \in] 0, M\left[, E_{T}=\{v \in\right.$ $C_{b}(] 0, T[\times \mathbb{R}) \mid \partial_{x} v \in C(] 0, T[\times \mathbb{R})$ and $\left.t^{1 / \lambda} \partial_{x} v \in C_{b}(] 0, T[\times \mathbb{R})\right\}$, endowed with its natural norm $\|v\|_{E_{T}}=\|v\|_{\infty}+\left\|t^{1 / \lambda} \partial_{x} v\right\|_{\infty}$. We define, thanks to Proposition 3.1, $\Psi_{T}: C_{b}(] 0, T[\times \mathbb{R}) \rightarrow C_{b}(] 0, T[\times \mathbb{R})$ by

$$
\Psi_{T}(v)(t, x)=K(t, \cdot) * v_{0}(x)+\int_{0}^{t} \partial_{x} K(t-s, \cdot) * F(s, \cdot, v(s, \cdot))(x) d s .
$$

Step 1: the first term of $\Psi_{T}(v)$ belongs to $E_{T}$. 
The estimate (2.2) allows to see, as in Step 1 of the proof of Proposition 3.1, that, by derivation and continuity under the integral sign, $K(t, \cdot) * v_{0}$ is derivable on $\mathbb{R}$ and that $(t, x) \in] 0, T\left[\times \mathbb{R} \rightarrow \partial_{x}\left(K(t, \cdot) * v_{0}\right)(x)=\partial_{x} K(t, \cdot) * v_{0}(x)\right.$ is continuous. By Young's inequalities and (2.4),

$$
\left\|\partial_{x}\left(K(t, \cdot) * v_{0}\right)\right\|_{C_{b}(\mathbb{R})} \leq \mathcal{K}_{1} t^{-1 / \lambda}\left\|v_{0}\right\|_{L^{\infty}(\mathbb{R})},
$$

which proves that $(t, x) \in] 0, T\left[\times \mathbb{R} \rightarrow K(t, \cdot) * v_{0}(x)\right.$ belongs to $E_{T}$.

Step 2: we prove that, if $v \in E_{T}$, the second term of $\Psi_{T}(v)$ belongs to $E_{T}$.

Define $H(t, x)=\int_{0}^{t} \partial_{x} K(t-s, \cdot) * F(s, \cdot, v(s, \cdot))(x) d s$. Let $\left.t \in\right] 0, T[$ and $s \in] 0, t[$. The function $x \in \mathbb{R} \rightarrow F(s, x, v(s, x))$ is in $C_{b}^{1}(\mathbb{R})$. We can thus differentiate under the integral sign to see that $\partial_{x} K(t-s, \cdot) * F(s, \cdot, v(s, \cdot))$ is $C^{1}$ with derivative $\partial_{x} K(t-s, \cdot) *\left(\partial_{x} F(s, \cdot, v(s, \cdot))+\partial_{\zeta} F(s, \cdot, v(s, \cdot)) \partial_{x} v(s, \cdot)\right)$. Moreover, for all $x \in \mathbb{R}$,

$$
\begin{gathered}
\left|\partial_{x} K(t-s, \cdot) *\left(\partial_{x} F(s, \cdot, v(s, \cdot))+\partial_{\zeta} F(s, \cdot, v(s, \cdot)) \partial_{x} v(s, \cdot)\right)(x)\right| \\
\quad \leq \frac{\mathcal{K}_{1}\left\|\partial_{x} F(\cdot, \cdot, v(\cdot, \cdot))\right\|_{\infty}}{(t-s)^{1 / \lambda}}+\frac{\mathcal{K}_{1}\left\|\partial_{\zeta} F(\cdot, \cdot, v(\cdot, \cdot))\right\|_{\infty}\|v\|_{E_{T}}}{s^{1 / \lambda}(t-s)^{1 / \lambda}} .
\end{gathered}
$$

This last function is integrable with respect to $s \in] 0, t[$, and we can thus apply the theorem of derivation under the integral sign to see that

$$
\begin{array}{rl}
\partial_{x} & H(t, x) \\
& =\int_{0}^{t} \partial_{x} K(t-s, \cdot) *\left(\partial_{x} F(s, \cdot, v(s, \cdot))+\partial_{\zeta} F(s, \cdot v(s, \cdot)) \partial_{x} v(s, \cdot)\right)(x) d s .
\end{array}
$$

If $\partial_{x} v$ was bounded, the continuity of $\partial_{x} H$ would be a consequence of Proposition 3.1. We thus approximate $\partial_{x} v$ by bounded functions to conclude. Take $0<\delta<T$ and define $w_{\delta} \in L^{\infty}(] 0, T[\times \mathbb{R})$ by

$$
w_{\delta}(t, x)=\partial_{x} F(t, x, v(t, x))+\partial_{\zeta} F(t, x, v(t, x)) \partial_{x} v(t, x) \mathbf{1}_{[\delta, T[}(t) .
$$

Denoting $A_{\delta}(t, x)=\int_{0}^{t} \partial_{x} K(t-s, \cdot) * w_{\delta}(s, \cdot)(x) d s$, (5.4) allows to see that, for all $\left.t_{0} \in\right] 0, T\left[, A_{\delta} \rightarrow \partial_{x} H\right.$ uniformly on $\left[t_{0}, T[\times \mathbb{R}\right.$ as $\delta \rightarrow 0$; since, by Proposition $3.1, A_{\delta}$ is continuous on $] 0, T\left[\times \mathbb{R}\right.$, we deduce that $\partial_{x} H$ is continuous on $] 0, T[\times \mathbb{R}$. Moreover, by (5.4) and (5.3) and the change of variable $s=t \tau$ in the integrals on ] $0, t[$, we have, for all $(t, x) \in] 0, T[\times \mathbb{R}$,

$$
\begin{aligned}
& \left|\partial_{x} H(t, x)\right| \\
& \quad \leq C_{0} \mathcal{K}_{1}\left(\left\|\partial_{x} F(\cdot, \cdot, v(\cdot, \cdot))\right\|_{\infty} t^{1-\frac{1}{\lambda}}+\left\|\partial_{\zeta} F(\cdot, \cdot, v(\cdot, \cdot))\right\|_{\infty}\|v\|_{E_{T}} t^{1-\frac{2}{\lambda}}\right)
\end{aligned}
$$

where $C_{0}=\max \left(\int_{0}^{1}(1-\tau)^{-1 / \lambda} d \tau, \int_{0}^{1} \tau^{-1 / \lambda}(1-\tau)^{-1 / \lambda} d \tau\right)$, which proves that $H \in E_{T}$. 
If $v$ is bounded by $R$, the properties of $F$ along with (5.2), (5.5) and (3.7), give

$$
\begin{aligned}
\left\|\Psi_{T}(v)\right\|_{E_{T}} \leq & \left\|v_{0}\right\|_{L^{\infty}(\mathbb{R})}+\frac{\lambda \mathcal{K}_{1}}{\lambda-1} T^{1-\frac{1}{\lambda}} \omega(R) \\
& +\mathcal{K}_{1}\left\|v_{0}\right\|_{L^{\infty}(\mathbb{R})}+C_{0} \mathcal{K}_{1} \omega(R)\left(T+T^{1-\frac{1}{\lambda}}\|v\|_{E_{T}}\right) .
\end{aligned}
$$

Step 3: fixed point.

We take, as in the proposition, $R=\left(2+\mathcal{K}_{1}\right) R_{0}$ and we denote, for $T>0$, $B_{T}(R)$ the closed ball in $E_{T}$ of center 0 and radius $R$. Let $T_{0}>0$ be such that

$$
\begin{aligned}
& R_{0}+\frac{\lambda \mathcal{K}_{1}}{\lambda-1} T_{0}^{1-\frac{1}{\lambda}} \omega(R)+\mathcal{K}_{1} R_{0}+C_{0} \mathcal{K}_{1} \omega(R)\left(T_{0}+T_{0}^{1-\frac{1}{\lambda}} R\right) \leq R \\
& \mathcal{K}_{1} \omega(R)\left(\frac{\lambda}{\lambda-1} T_{0}^{1-\frac{1}{\lambda}}+\frac{\lambda}{\lambda-1} T_{0}+C_{0} R T_{0}^{1-\frac{1}{\lambda}}+C_{0} T_{0}^{1-\frac{1}{\lambda}}\right)<1
\end{aligned}
$$

(by definition of $R$, such a $T_{0}$ exists and only depends on $\left(R_{0}, \omega\right)$ ).

Let $T=\inf \left(M, T_{0}\right)$. Take $v_{0} \in L^{\infty}(\mathbb{R})$ bounded by $R_{0}$. Thanks to $(5.6)$ and (5.7), $\Psi_{T}$ sends $B_{T}(R)$ into $B_{T}(R)$. Let $(u, v) \in B_{T}(R)$. $u$ and $v$ are bounded by $R$ and we have thus, for all $(t, x) \in] 0, T[\times \mathbb{R}$, by (2.4) and the properties of $F$,

$$
\left|\Psi_{T}(u)(t, s)-\Psi_{T}(v)(t, x)\right| \leq \mathcal{K}_{1} \frac{\lambda}{\lambda-1} T^{1-\frac{1}{\lambda}} \omega(R)\|u-v\|_{\infty} .
$$

By (5.4) and the properties of $F$, we also have, for all $(t, x) \in] 0, T[\times \mathbb{R}$,

$$
\begin{aligned}
& t^{1 / \lambda}\left|\partial_{x} \Psi_{T}(u)(t, x)-\partial_{x} \Psi_{T}(v)(t, x)\right| \\
& \quad \leq \mathcal{K}_{1} \omega(R)\left(\frac{\lambda}{\lambda-1} T+C_{0} T^{1-\frac{1}{\lambda}}\|u\|_{E_{T}}+C_{0} T^{1-\frac{1}{\lambda}}\right)\|u-v\|_{E_{T}} .
\end{aligned}
$$

The properties (5.9), (5.10) and (5.8) ensure that $\Psi_{T}$ is contracting on $B_{T}(R)$. Therefore, $\Psi_{T}$ has a unique fixed point $v$ in $B_{T}(R) ; v$ is a continuous and bounded solution to (5.1) such that $\partial_{x} v$ exists and is continuous on $] 0, T[\times \mathbb{R}$. Moreover, since $v \in B_{T}(R)$, we have, for all $\left.a \in\right] 0, T[$ and all $(t, x) \in] a, T\left[\times \mathbb{R},\left|\partial_{x} v(t, x)\right| \leq\right.$ $t^{-1 / \lambda}\|v\|_{E_{T}} \leq a^{-1 / \lambda} R$, which is the estimate on $\partial_{x} v$ stated in the proposition.

The inequalities (5.9) and (5.8) ensure that $\Psi_{T}$ is contracting on the ball in $C_{b}(] 0, T[\times \mathbb{R})$ of center 0 and radius $R$. Thus, $\Psi_{T}$ can have only one fixed point in this ball, which is the uniqueness result of the proposition.

Theorem 5.1 Let $u_{0} \in L^{\infty}(\mathbb{R})$ and $T>0$ or $T=\infty$. If $u$ is a solution to (1.1) on $] 0, T$ [ in the sense of Definition 3.1, then $u$ is indefinitely derivable with respect to $x$. Moreover, for all $n \geq 0$ and all $\left.t_{0} \in\right] 0, T[$, we have

i) $\partial_{x}^{n} u \in C_{b}(] t_{0}, T[\times \mathbb{R})$, 
ii) for all $t \in] 0, T-t_{0}[$,

$$
\partial_{x}^{n} u\left(t_{0}+t, \cdot\right)=K(t, \cdot) * \partial_{x}^{n} u\left(t_{0}, \cdot\right)-\int_{0}^{t} \partial_{x} K(t-s, \cdot) * \partial_{x}^{n}\left(f\left(u\left(t_{0}+s, \cdot\right)\right)\right) d s
$$

iii) if $R \geq\|u\|_{C_{b}(] 0, T[\times \mathbb{R})}$, there exists $C$ only depending on $\left(R, t_{0}, n\right)$ such that $\left\|\partial_{x}^{n} u\right\|_{C_{b}(] t_{0}, T[\times \mathbb{R})} \leq C$.

\section{Proof of Theorem 5.1}

We prove, by induction on $n$, that : $u$ has spatial derivatives of order up to $n$ which are continuous and bounded by $C\left(R, t_{0}, n\right)$ on $] t_{0}, T\left[\times \mathbb{R}\right.$ for all $\left.t_{0} \in\right] 0, T[$, item ii) is satisfied on $] 0, T-t_{0}\left[\times \mathbb{R}\right.$ for all $\left.t_{0} \in\right] 0, T[$ and

$$
\partial_{x}^{n}(f(u))=U_{n}+\left(1-\delta_{n, 0}\right) f^{\prime}(u) \partial_{x}^{n} u+\delta_{n, 0} f(u),
$$

where $\delta_{n, 0}$ is Krönecker's symbol, $U_{0}=0$ and, if $n \geq 1, U_{n}=G_{n}\left(\left(\partial_{x}^{k} u\right)_{k \leq n-1}\right)$ with $G_{n}$ regular.

The validity of the property at the rank $n=0$ is a consequence of Corollary 3.1. We suppose the induction hypothesis true up to a rank $n \geq 0$, and we prove it for the rank $n+1$.

Let $\left.b_{0} \in\right] 0, T[$. Take $b \in] b_{0}, T[$ and define $F:(t, x, \zeta):] 0, T-b[\times \mathbb{R} \times \mathbb{R} \rightarrow$ $-U_{n}(b+t, x)-\left(1-\delta_{n, 0}\right) f^{\prime}(u)(b+t, x) \zeta-\delta_{n, 0} f(\zeta)$. The function $F$ satisfies the hypotheses of Proposition 5.1, with (by induction hypothesis) $\omega$ only depending on $\left(R, b_{0}, n\right)$; we also have $\left\|\partial_{x}^{n} u\right\|_{C_{b}\left(b_{0}, T[\times \mathbb{R})\right.} \leq R_{0}$ where $R_{0}$ only depends on $\left(R, b_{0}, n\right)$. Let $T_{0}$ only depending on $\left(R_{0}, \omega\right)$ (i.e. on $\left.\left(R, b_{0}, n\right)\right)$ be given by Proposition 5.1.

By induction hypothesis, $\partial_{x}^{n} u(b+\cdot, \cdot)$ is continuous and bounded by $R_{0} \leq(2+$ $\left.\mathcal{K}_{1}\right) R_{0}$ and satisfies $(5.1)$ on $] 0, T-b\left[\times \mathbb{R}\right.$ for the preceding $F$ and with $v_{0}=\partial_{x}^{n} u(b, \cdot)$ bounded by $R_{0}$. Proposition 5.1 shows thus that $\partial_{x}^{n+1} u$ exists and is continuous and bounded by $\left(2+\mathcal{K}_{1}\right) R_{0} a^{-1 / \lambda}$ on $] b+a, \inf \left(T, b+T_{0}\right)[\times \mathbb{R}$; this is true for all $b \in] b_{0}, T[$ and all $a \in] 0, \inf \left(T-b, T_{0}\right)\left[\right.$. Since $T_{0}$ does not depend on $a$ or $b$, taking $\left.t_{0} \in\right] 0, T\left[, b_{0}=t_{0} / 2\right.$ and $a=\inf \left(t_{0} / 2, T_{0} / 2\right)<T-b_{0}$, we notice that the intervals \{]$b+a, \inf \left(T, b+T_{0}\right)[, b \in] b_{0}, T-a[\}$ cover $] b_{0}+a, T[\supset] t_{0}, T[$ and we deduce that $\partial_{x}^{n+1} u$ has the regularity and satisfies the estimates we wanted to obtain.

Let us prove the formula for $\partial_{x}^{n+1} u$. By induction hypothesis,

$$
\partial_{x}^{n} u\left(t_{0}+t, \cdot\right)=K(t, \cdot) * \partial_{x}^{n} u\left(t_{0}, \cdot\right)-\int_{0}^{t} \partial_{x} K(t-s, \cdot) * \partial_{x}^{n}\left(f\left(u\left(t_{0}+\cdot\right)\right)\right) d s .
$$

But we have just proved that $\partial_{x}^{n} u\left(t_{0}, \cdot\right) \in C_{b}^{1}(\mathbb{R})$; thus, we can write

$$
\partial_{x}\left(K(t, \cdot) * \partial_{x}^{n} u\left(t_{0}, \cdot\right)\right)=K(t, \cdot) * \partial_{x}^{n+1} u\left(t_{0}, \cdot\right) .
$$

The function $(t, x) \in] 0, T-t_{0}\left[\times \mathbb{R} \rightarrow \partial_{x}^{n}(f(u))\left(t_{0}+t, x\right)\right.$ and its first spatial derivative are continuous and bounded on $] 0, T-t_{0}[\times \mathbb{R}$. The reasoning of Step 2 in the 
proof of Proposition 5.1 (with $\partial_{x}^{n}(f(u))\left(t_{0}+\cdot, \cdot\right)$ instead of $F(\cdot, \cdot, v(\cdot, \cdot))$ ) allows to compute the spatial derivative of the last term in (5.12) by derivation under the integral sign, and, thanks to (5.13), proves item ii) for $\partial_{x}^{n+1} u$.

Property (5.11) for the derivative of order $n+1$ simply comes from the derivation of this formula at rank $n$, and the induction is complete.

\subsection{Temporal regularity}

\subsubsection{Preliminary: about the definition of $g$}

The operator $g$ has been formally defined by $\mathcal{F}(g[v])(\xi)=|\xi|^{\lambda} \mathcal{F}(v)(\xi)$; it can be shown that this definition makes sense for bounded functions, but we will not need it and we prefer to give here a simple formula for $g[v]$ which defines this operator on $C_{b}^{\infty}(\mathbb{R})$.

Proposition 5.2 There exists $\left(g_{1}, g_{2}\right) \in\left(L^{1}(\mathbb{R})\right)^{2}$ such that, for all $v \in \mathcal{S}(\mathbb{R})$, $g[v]=g_{1} * v+g_{2} * v^{(4)}$. This formula allows thus to define $g[v]$ for $v \in C_{b}^{\infty}(\mathbb{R})$ (and this definition does not depend on the choice of $g_{1}$ and $g_{2}$ as above).

\section{Proof of Proposition 5.2}

Let $\chi \in C_{c}^{\infty}(\mathbb{R})$ be even and equal to 1 on a neighborhood of 0 . By linearity of $\mathcal{F}^{-1}$, if $v \in \mathcal{S}(\mathbb{R})$,

$$
g[v]=\mathcal{F}^{-1}\left(|\cdot|{ }^{\lambda} \chi \mathcal{F}(v)\right)+\mathcal{F}^{-1}\left(|\cdot|^{\lambda}(1-\chi) \mathcal{F}(v)\right)
$$

(since $\mathcal{F}(v) \in \mathcal{S}(\mathbb{R})$, all these terms are well-defined as inverse Fourier transforms of integrable functions).

Let $h_{1}: \xi \in \mathbb{R} \rightarrow|\xi|^{\lambda} \chi(\xi)$. The function $h_{1}$ is $C^{1}$ on $\mathbb{R}, C^{2}$ outside 0 and its first two derivatives are integrable on $\mathbb{R}$. We deduce, as in the proof of $(2.2)$, that $\mathcal{F}^{-1}\left(h_{1}\right)(x)=\mathcal{O}\left(1 /\left(1+|x|^{2}\right)\right)$ on $\mathbb{R}$. Hence, $\mathcal{F}^{-1}\left(h_{1}\right) \in L^{1}(\mathbb{R})$ and we can write $\mathcal{F}\left(\mathcal{F}^{-1}\left(h_{1}\right) * v\right)=h_{1} \mathcal{F}(v)$, that is to say $\mathcal{F}^{-1}\left(h_{1} \mathcal{F}(v)\right)=\mathcal{F}^{-1}\left(h_{1}\right) * v$.

Let $h_{2}: \xi \in \mathbb{R} \rightarrow|\xi|^{\lambda}(1-\chi(\xi))$; the function $h_{2}^{\star}: \xi \in \mathbb{R} \rightarrow(2 i \pi \xi)^{-4} h_{2}(\xi)$ is $C^{\infty}$ and all its derivatives are integrable on $\mathbb{R}$ (the $p$-th derivative of $h_{2}^{\star}$ behaves, on a neighborhood of the infinity, as $|\xi|^{-4-p+\lambda}$ and $-4+\lambda<-1$ since $\lambda \leq 2$ ); thus, $\mathcal{F}^{-1}\left(h_{2}^{\star}\right) \in L^{1}(\mathbb{R})$ and

$$
\mathcal{F}\left(\mathcal{F}^{-1}\left(h_{2}^{\star}\right) * v^{(4)}\right)(\xi)=h_{2}^{\star}(\xi) \mathcal{F}\left(v^{(4)}\right)(\xi)=(2 i \pi \xi)^{4} h_{2}^{\star}(\xi) \mathcal{F}(v)(\xi)=h_{2}(\xi) \mathcal{F}(v)(\xi),
$$

that is to say $\mathcal{F}^{-1}\left(h_{2} \mathcal{F}(v)\right)=\mathcal{F}^{-1}\left(h_{2}^{\star}\right) * v^{(4)}$.

Identity (5.14) gives therefore $g[v]=g_{1} * v+g_{2} * v^{(4)}$, where $g_{1}=\mathcal{F}^{-1}\left(h_{1}\right)$ and $g_{2}=\mathcal{F}^{-1}\left(h_{2}^{\star}\right)$ are integrable on $\mathbb{R}$ (notice also that, since $\chi$ is even, $h_{1}$ and $h_{2}^{\star}$ are also even and real-valued, so that $g_{1}$ and $g_{2}$ are real-valued).

To prove that, if $v \in C_{b}^{\infty}(\mathbb{R})$, the definition of $g[v]$ by this formula does not depend on the choice of $g_{1}$ and $g_{2}$, we approximate $v$ and its derivatives by functions in $C_{c}^{\infty}(\mathbb{R})$; this will be of no use to us in the sequel, so we do not detail this step. 
The following proposition is quite natural, since $K$ is the kernel associated to $g$. But the reasoning followed to obtain $K$ was formal, so we must prove this result.

Proposition 5.3 If $v \in C_{b}^{\infty}(\mathbb{R})$ then, for all $\left.x \in \mathbb{R}, t \in\right] 0, \infty[\rightarrow K(t, \cdot) * v(x)$ is $C^{1}$. Moreover, for all $t>0$ and all $x \in \mathbb{R}$, we have $\frac{d}{d t}(K(\cdot, \cdot) * v(x))(t)=$ $-g[K(t, \cdot) * v](x)$.

\section{Proof of Proposition 5.3}

We notice that $g[K(t, \cdot) * v]$ makes sense since $K(t, \cdot) * v \in C_{b}^{\infty}(\mathbb{R})$.

Suppose first that $v \in \mathcal{S}(\mathbb{R})$. The functions $K(t, \cdot)$ and $v$ are integrable on $\mathbb{R}$, so, by definition of $K, K(t, \cdot) * v=\mathcal{F}^{-1}\left(e^{-t|\cdot|^{\lambda}} \mathcal{F}(v)\right)$. A derivation under the integral sign shows that $t \in] 0, \infty\left[\rightarrow K(t, \cdot) * v(x)\right.$ is $C^{1}$ and that, for all $t>0$ and all $x \in \mathbb{R}$,

$$
\frac{d}{d t}(K(\cdot, \cdot) * v(x))(t)=-\mathcal{F}^{-1}\left(|\cdot|^{\lambda} e^{-t|\cdot|^{\lambda}} \mathcal{F}(v)\right)(x) .
$$

Taking $g_{1}$ and $g_{2}$ as in the proof of Proposition 5.2, we can check, since $K(t, \cdot)$ and all the derivatives of $v$ are integrable, that $\mathcal{F}(g[K(t, \cdot) * v])=|\cdot|{ }^{\lambda} e^{-t|\cdot|^{\lambda}} \mathcal{F}(v)$, that is to say $g[K(t, \cdot) * v]=\mathcal{F}^{-1}\left(|\cdot|^{\lambda} e^{-t|\cdot|^{\lambda}} \mathcal{F}(v)\right)$ and (5.15) concludes the proof if $v \in \mathcal{S}(\mathbb{R})$

Take now $v \in C_{b}^{\infty}(\mathbb{R})$. We can find a sequence $\left(v_{n}\right)_{n>1} \in \mathcal{S}(\mathbb{R})$ whose derivatives are bounded in $L^{\infty}(\mathbb{R})$ and converge to the corresponding derivatives of $v$.

Let $x \in \mathbb{R}$; define $\left.F_{n}: t \in\right] 0, \infty\left[\rightarrow K(t, \cdot) * v_{n}(x)\right.$ and $\left.F: t \in\right] 0, \infty[\rightarrow K(t, \cdot) *$ $v(x)$. By the convergence of $\left(v_{n}\right)_{n \geq 1}$ and the dominated convergence theorem, we see that $\left(F_{n}\right)_{n \geq 1}$ converges to $F$ on $] 0, \infty\left[\right.$ and is bounded in $L^{\infty}(] 0, \infty[)$. Therefore, the convergence is also true in the sense of the distributions on $] 0, \infty[$ and we have $F_{n}^{\prime} \rightarrow F^{\prime}$ in $\mathcal{D}^{\prime}(] 0, \infty[)$.

But, since $v_{n} \in \mathcal{S}(\mathbb{R})$, we have seen that $F_{n}$ is $C^{1}$ and that $F_{n}^{\prime}(t)=-g[K(t, \cdot) *$ $\left.v_{n}\right](x)=-g_{1} * K(t, \cdot) * v_{n}(x)-g_{2} * K(t, \cdot) * v_{n}^{(4)}(x)$. Hence, $\left(F_{n}^{\prime}\right)_{n>1}$ converges to $-g_{1} * K(t, \cdot) * v(x)-g_{2} * K(t, \cdot) * v^{(4)}(x)=-g[K(t, \cdot) * v](x)$ and is bounded in $L^{\infty}(] 0, \infty[)$, which proves that $F_{n}^{\prime} \rightarrow-g[K(t, \cdot) * v](x)$ in $\mathcal{D}^{\prime}(] 0, \infty[)$.

Identifying the limits of the derivatives of $F_{n}$, we find $F^{\prime}(t)=-g[K(t, \cdot) * v](x)$ in $\mathcal{D}^{\prime}(] 0, \infty[) ;$ since $\left.t \in\right] 0, \infty\left[\rightarrow g[K(t, \cdot) * v](x)=g_{1} * K(t, \cdot) * v(x)+g_{2} * K(t, \cdot) * v^{(4)}(x)\right.$ is continuous (Proposition 3.1 with $u_{0}=g_{1} * v$ or $u_{0}=g_{2} * v^{(4)}$ ), we deduce that $F: t \in] 0, \infty\left[\rightarrow K(t, \cdot) * v(x)\right.$ is in fact $C^{1}$ on $] 0, \infty[$, which concludes the proof.

\subsubsection{Proof of the temporal regularity}

Lemma 5.1 Let $u_{0} \in L^{\infty}(\mathbb{R})$ and $T>0$ or $T=\infty$. If $u$ is a solution to (1.1) on $] 0, T[$ in the sense of Definition 3.1, then $u$ is derivable with respect to $t$ and $\partial_{t} u+\partial_{x}(f(u))+g[u]=0$ on $] 0, T[\times \mathbb{R}$. 


\section{Proof of Lemma 5.1}

We can suppose that $T$ is finite. Let $\left.t_{0}>0, t \in\right] t_{0}, T[$ and $s \in] 0, t[$. Using (3.4) (and an equivalent estimate for $\partial_{x} K$, obtained thanks to $\left.(2.2)\right)$, since $f\left(u\left(t_{0}+s, \cdot\right)\right)$ is in $C_{b}^{1}(\mathbb{R})$, we see that $\partial_{x} K(t-s, \cdot) * f\left(u\left(t_{0}+s, \cdot\right)\right)=K(t-s, \cdot) * \partial_{x}\left(f\left(u\left(t_{0}+s, \cdot\right)\right)\right)$.

Defining $v:(t, x) \in] 0, T-t_{0}\left[\times \mathbb{R} \rightarrow-\partial_{x}(f(u))\left(t_{0}+t, x\right) \in \mathbb{R}\right.$ (which is continuous and bounded, and has all its spatial derivatives continuous and bounded - see Theorem 5.1), we write, by (3.8),

$$
u\left(t_{0}+t, x\right)=K(t, \cdot) * u\left(t_{0}, \cdot\right)+\int_{0}^{t} K(t-s, \cdot) * v(s, \cdot)(x) d s .
$$

Since $u\left(t_{0}, \cdot\right) \in C_{b}^{\infty}(\mathbb{R})$, Proposition 5.3 shows that $\left.(t, x) \in\right] 0, T-t_{0}[\times \mathbb{R} \rightarrow$ $K(t, \cdot) * u\left(t_{0}, \cdot\right)(x)$ is derivable with respect to $t$, and has $-g\left[K(t, \cdot) * u\left(t_{0}, \cdot\right)\right](x)$ as derivative.

Proving the derivability of the second term of the right-hand side of (5.16) is more troublesome (because $K(t-s, \cdot)$ explodes as $s \rightarrow t$ ). Fix $x \in \mathbb{R}$ and $\left.\delta_{0} \in\right] 0, T-t_{0}[$. Let $\delta \in] 0, \delta_{0}[$ and, for $t \in] \delta_{0}, T-t_{0}\left[, H_{\delta}(t)=\int_{0}^{t-\delta} K(t-s, \cdot) *\right.$ $v(s, \cdot)(x) d s$. Also denote $H(t)=\int_{0}^{t} K(t-s, \cdot) * v(s, \cdot)(x) d s$. We have $\mid H_{\delta}(t)-$ $H(t) \mid \leq \delta\|v\|_{C_{b}(] 0, T-t_{0}[\times \mathbb{R})} d s$, so that $H_{\delta} \rightarrow H$ uniformly on $] \delta_{0}, T-t_{0}[$ as $\delta \rightarrow 0$.

The function $\phi:(t, s) \in\left\{\left(t^{\prime}, s^{\prime}\right) \in\right] \delta, T-t_{0}[\times] 0, T-t_{0}\left[\mid s^{\prime}<t^{\prime}-\delta / 2\right\} \rightarrow$ $K(t-s, \cdot) * v(s, \cdot)(x)$ is continuous (Lemma $2.2 \mathrm{ii})$-a)) and bounded. By Proposition $5.3, \phi$ is derivable with respect to $t$ and $\partial_{t} \phi(t, s)=-g[K(t-s, \cdot) * v(s, \cdot)](x)=$ $-g_{1} * K(t-s, \cdot) * v(s, \cdot)(x)-g_{2} * K(t-s, \cdot) * \partial_{x}^{4} v(s, \cdot)(x)$; this formula and Lemma 2.2 ii)-a) show that $\partial_{t} \phi$ is continuous and bounded (because, by continuity under the integral sign, $(s, x) \rightarrow g_{1} * v(s, \cdot)(x)$ and $(s, x) \rightarrow g_{2} * \partial_{x}^{4} v(s, \cdot)(x)$ are continuous and bounded on $] 0, T-t_{0}[\times \mathbb{R})$. These properties allow to prove that $H_{\delta}$ is $C^{1}$ on ]$\delta_{0}, T-t_{0}[$ and that

$$
H_{\delta}^{\prime}(t)=K(\delta, \cdot) * v(t-\delta, \cdot)(x)-\int_{0}^{t-\delta} g[K(t-s, \cdot) * v(s, \cdot)](x) d s .
$$

The function $(s, x) \in] 0, t\left[\times \mathbb{R} \rightarrow g[K(t-s, \cdot) * v(s, \cdot)](x)=g_{1} * K(t-s, \cdot) *\right.$ $v(s, \cdot)(x)+g_{2} * K(t-s, \cdot) * \partial_{x}^{4} v(s, \cdot)(x)$ is continuous and bounded (Lemma 2.2 ii)-a)); thus, by Lemma $2.2 \mathrm{ii})$-b), we see that $H_{\delta}^{\prime}$ converges on $] \delta_{0}, T-t_{0}$ [ to

$$
F: t \in] 0, T-t_{0}\left[\rightarrow v(t, x)-\int_{0}^{t} g[K(t-s, \cdot) * v(s, \cdot)](x) d s\right.
$$

while remaining bounded in $L^{\infty}(] \delta_{0}, T-t_{0}[)$. Since $H_{\delta}$ uniformly converges on ]$\delta_{0}, T-t_{0}\left[\right.$ to $H$, we deduce that $H^{\prime}=F$ in $\mathcal{D}^{\prime}(] \delta_{0}, T-t_{0}[)$. Since $g[K(t-s, \cdot) *$ $v(s, \cdot)]=g_{1} * K(t-s, \cdot) * v(s, \cdot)+g_{2} * K(t-s, \cdot) * \partial_{x}^{4} v(s, \cdot)$, the same reasoning as in Step 2 of the proof of Proposition 3.1 (with $K$ instead of $\partial_{x} K$ and $(t, x) \rightarrow g_{1} * v(t, \cdot)(x)$ 
or $(t, x) \rightarrow g_{2} * \partial_{x}^{4} v(t, \cdot)(x)$ instead of $\left.v\right)$ shows that $F$ is in fact continuous. Hence, $\delta_{0}$ being arbitrary, $H$ is $C^{1}$ on $] 0, T-t_{0}\left[\right.$ and $H^{\prime}=F$.

Coming back to $(5.16)$, we see that $u\left(t_{0}+\cdot, \cdot\right)$ is derivable with respect to $t$ on ] $0, T-t_{0}[\times \mathbb{R}$ and that

$$
\begin{aligned}
& \partial_{t} u\left(t_{0}+t, x\right)=-g\left[K(t, \cdot) * u\left(t_{0}, \cdot\right)\right](x)-\partial_{x}(f(u))\left(t_{0}+t, x\right) \\
& \quad-\int_{0}^{t} g_{1} * K(t-s, \cdot) * v(s, \cdot)(x)+g_{2} * K(t-s, \cdot) * \partial_{x}^{4} v(s, \cdot)(x) d s .
\end{aligned}
$$

The time $t_{0}$ being arbitrary, this gives the temporal derivability of $u$ on $] 0, T[\times \mathbb{R}$. We now prove that the right-hand side of (5.17) is $-\partial_{x}(f(u))-g[u]$.

Let $t \in] 0, T-t_{0}[$. By Fubini, we have

$$
\begin{aligned}
& \int_{0}^{t} g_{1} * K(t-s, \cdot) * v(s, \cdot)+g_{2} * K(t-s, \cdot) * \partial_{x}^{4} v(s, \cdot) d s \\
& =g_{1} *\left[\int_{0}^{t} K(t-s, \cdot) * v(s, \cdot) d s\right]+g_{2} *\left[\int_{0}^{t} K(t-s, \cdot) * \partial_{x}^{4} v(s, \cdot) d s\right] .
\end{aligned}
$$

With the same reasoning as in Step 2 of the proof of Proposition 5.1 (with $K$ instead of $\partial_{x} K$ and $v$ instead of $F(\cdot, \cdot, v(\cdot, \cdot))$ ), we prove by induction that $(t, x) \in$ ] $0, T-t_{0}\left[\times \mathbb{R} \rightarrow \int_{0}^{t} K(t-s, \cdot) * v(s, \cdot)(x) d s\right.$ is indefinitely derivable with respect to $x$, has all its spatial derivatives continuous and bounded on $] 0, T-t_{0}[\times \mathbb{R}$ and satisfies, for all $m \geq 0$,

$$
\partial_{x}^{m}\left(\int_{0}^{t} K(t-s, \cdot) * v(s, \cdot) d s\right)=\int_{0}^{t} K(t-s, \cdot) * \partial_{x}^{m} v(s, \cdot) d s .
$$

Thus, by (5.18),

$$
\begin{aligned}
& \int_{0}^{t} g_{1} * K(t-s, \cdot) * v(s, \cdot)+g_{2} * K(t-s, \cdot) * \partial_{x}^{4} v(s, \cdot) d s \\
& \quad=g_{1} *\left[\int_{0}^{t} K(t-s, \cdot) * v(s, \cdot) d s\right]+g_{2} * \partial_{x}^{4}\left[\int_{0}^{t} K(t-s, \cdot) * v(s, \cdot) d s\right] \\
& \quad=g\left[\int_{0}^{t} K(t-s, \cdot) * v(s, \cdot) d s\right] .
\end{aligned}
$$

This equation, combined with (5.17) and (5.16), shows that $u$ satisfies $\partial_{t} u+$ $\partial_{x}(f(u))+g[u]=0$ on $] t_{0}, T\left[\times \mathbb{R}\right.$ for all $t_{0}>0$, which concludes the proof.

Item i) of Theorem 1.1 is a direct consequence of Theorem 5.1, Lemma 5.1 and Proposition 5.2 (as well as the theorem of continuity under the integral sign and Young's inequalities which show that, if $v \in C_{b}(] t_{0}, T[\times \mathbb{R})$ and $w \in L^{1}(\mathbb{R})$, then $(t, x) \in] t_{0}, T[\times \mathbb{R} \rightarrow w * v(t, \cdot)(x)$ is continuous and bounded). 


\section{$6 \quad L^{\infty}$ estimate and global existence}

We construct here a solution to (1.1) on $] 0, \infty\left[\right.$ which is bounded by $\left\|u_{0}\right\|_{L^{\infty}(\mathbb{R})}$ and satisfies the maximum principle, thus concluding the proof of Theorem 1.1.

We assume, in the three following subsections, that $u_{0} \in C_{c}^{\infty}(\mathbb{R})$ (in fact, we just need $\left.u_{0} \in L^{1}(\mathbb{R}) \cap B V(\mathbb{R})\right)$.

\subsection{Construction of an approximate solution by a splitting method}

Let $\delta>0$. We construct, by induction, a function $u^{\delta}:[0, \infty[\times \mathbb{R} \rightarrow \mathbb{R}$ the following way: we let $u^{\delta}(0, \cdot)=u_{0}$ and, for all $n \geq 0$, we define

- $u^{\delta}$ on $\left.] 2 n \delta,(2 n+1) \delta\right] \times \mathbb{R}$ as the solution to $\partial_{t} u^{\delta}+2 g\left[u^{\delta}\right]=0\left(^{1}\right)$ with initial condition $u^{\delta}(2 n \delta, \cdot)$, that is to say $u^{\delta}(t, x)=K(2(t-2 n \delta), \cdot) * u^{\delta}(2 n \delta, \cdot)(x)$ for $(t, x) \in] 2 n \delta,(2 n+1) \delta] \times \mathbb{R}$.

- $u^{\delta}$ on $\left.](2 n+1) \delta, 2(n+1) \delta\right] \times \mathbb{R}$ as the (entropy) solution to $\partial_{t} u^{\delta}+2 \partial_{x}\left(f\left(u^{\delta}\right)\right)=$ 0 with initial condition $u^{\delta}((2 n+1) \delta, \cdot)$.

Since $\|K(t, \cdot)\|_{L^{1}(\mathbb{R})}=1$ for all $t>0$, the regularizing operator does not increase the $L^{\infty}$ norm (in fact, $K$ being nonnegative, the maximum principle is satisfied), the $L^{1}$ norm and the $B V$ semi-norm; it is a well-known result that the hyperbolic operator has the same properties. Moreover, the solutions to both equations are continuous with values in $L^{1}(\mathbb{R})$ (this is what states Lemma 2.2-i) for the regularizing equation). We have therefore defined $u^{\delta} \in C\left(\left[0, \infty\left[; L^{1}(\mathbb{R})\right)\right.\right.$ such that $u^{\delta}(0, \cdot)=u_{0}$,

$$
\begin{gathered}
\forall t \geq 0,\left\|u^{\delta}(t, \cdot)\right\|_{L^{\infty}(\mathbb{R})} \leq\left\|u_{0}\right\|_{L^{\infty}(\mathbb{R})},\left\|u^{\delta}(t, \cdot)\right\|_{L^{1}(\mathbb{R})} \leq\left\|u_{0}\right\|_{L^{1}(\mathbb{R})} \\
\text { and }\left|u^{\delta}(t, \cdot)\right|_{B V(\mathbb{R})} \leq\left\|u_{0}^{\prime}\right\|_{L^{1}(\mathbb{R})},
\end{gathered}
$$

(in fact, $u^{\delta}$ takes its values between the minimum and maximum values of $u_{0}$ ) and, for all $n \geq 0$,

$$
\begin{aligned}
& \left.\left.u^{\delta}(t, \cdot)=K(2(t-2 n \delta), \cdot) * u^{\delta}(2 n \delta, \cdot) \text { for all } t \in\right] 2 n \delta,(2 n+1) \delta\right] \\
& \left.\left.u^{\delta} \text { satisfies } \partial_{t} u^{\delta}+2 \partial_{x}\left(f\left(u^{\delta}\right)\right)=0 \text { on }\right](2 n+1) \delta, 2(n+1) \delta\right] \times \mathbb{R} .
\end{aligned}
$$

By (2.2) (which also gives, through (2.1), estimates on the time derivatives of $K)$ and the fact that $u^{\delta}(2 n \delta, \cdot) \in L^{\infty}(\mathbb{R})$, we see that, for all $n \geq 0, u^{\delta}$ is $C^{\infty}$ on ] $2 n \delta,(2 n+1) \delta] \times \mathbb{R}$. Moreover,

$$
\left\|\partial_{x} u^{\delta}((2 n+1) \delta, \cdot)\right\|_{\infty}=\left\|\partial_{x} K(2 \delta, \cdot) * u^{\delta}(2 n \delta, \cdot)\right\|_{\infty} \leq \mathcal{K}_{1}\left\|u_{0}\right\|_{\infty}(2 \delta)^{-1 / \lambda} .
$$

\footnotetext{
${ }^{1}$ The factor 2 comes from the fact that we solve the regularizing equation (and the hyperbolic equation) on half of the total time, so we must give it twice more weight.
} 
Hence, the time of regularity of $u^{\delta}$ on $\left.](2 n+1) \delta, 2(n+1) \delta\right] \times \mathbb{R}$ is at least

$$
T^{*} \geq \frac{1}{2\left\|f^{\prime \prime}\left(u^{\delta}((2 n+1) \delta)\right) \partial_{x} u^{\delta}((2 n+1) \delta)\right\|_{L^{\infty}(\mathbb{R})}} \geq C_{0} \delta^{1 / \lambda}
$$

where $C_{0}$ does not depend on $\delta$ or $n$ (we have used (6.1) to bound $f^{\prime \prime}\left(u^{\delta}((2 n+1) \delta)\right)$ ). For $\delta$ small enough, this time of regularity is thus greater than $\delta$.

The parameter $\delta$ being destined to tend to 0 , we can always suppose that it is small enough (let us say $\delta \leq \delta_{0}$ ) in order that $u^{\delta}$ is regular on $\left.] 2 n \delta,(2 n+1) \delta\right] \times \mathbb{R}$ and on $[(2 n+1) \delta, 2(n+1) \delta] \times \mathbb{R}$ for all $n \geq 0$ (the $B V$ estimate of (6.1) turns then into a $L^{1}$ estimate on the first spatial derivative).

Remark 6.1 It is also possible to construct $u^{\delta}$ via a classical splitting method, i.e. to solve the regularizing equation (without the factor 2 ) on $[k \delta,(k+1) \delta]$ and then use the value thus obtained at $t=(k+1) \delta$ to solve the hyperbolic equation (still without the factor 2 ) on $[k \delta,(k+1) \delta]$ once again (and not on $[(k+1) \delta,(k+2) \delta]$ ). All the following reasoning can be done with such a construction; however, since the function thus defined is not continuous on $[0, \infty[$, more work is to be done.

\subsection{Compactness result on the sequence $\left(u^{\delta}\right)_{\delta>0}$}

Proposition 6.1 For all compact subset $Q$ of $\mathbb{R}$ and all $\left.\left.T>0,\left\{u^{\delta}, \delta \in\right] 0, \delta_{0}\right]\right\}$ is relatively compact in $C\left([0, T] ; L^{1}(Q)\right)$.

\section{Proof of Proposition 6.1}

Let $Q$ be a compact subset of $\mathbb{R}$ and $T>0$. For all $t \in[0, T]$, we have, by (6.1), $\left\|u^{\delta}(t)\right\|_{L^{1}(\mathbb{R}) \cap B V(\mathbb{R})} \leq\left\|u_{0}\right\|_{W^{1,1}(\mathbb{R})}$ (we omit the space variable in $u^{\delta}$ ); thus, by Helly's Theorem, $\left.\left.\left\{u^{\delta}(t, \cdot), \delta \in\right] 0, \delta_{0}\right]\right\}$ is relatively compact in $L^{1}(Q)$

We will prove the equicontinuity of $\left.\left.\left\{u^{\delta}, \delta \in\right] 0, \delta_{0}\right]\right\}$ in $C\left(\left[0, \infty\left[; L^{1}(\mathbb{R})\right)\right.\right.$; this implies the equicontinuity in $C\left([0, T] ; L^{1}(Q)\right)$ and, thanks to Ascoli-Arzela's theorem, concludes the proof of the proposition.

It is classical that the solution to an hyperbolic equation is lipschitz-continuous $\left[0, \infty\left[\rightarrow L^{1}(\mathbb{R})\right.\right.$. Thanks to $(6.1)$, we see that the lipschitz constant of $u^{\delta}$ on $[(2 n+$ 1) $\delta, 2(n+1) \delta]$ does not depend on $\delta$ or $n \geq 0$ : there exists $C_{0}$ such that, for all $\left.\delta \in] 0, \delta_{0}\right]$, for all $n \geq 0$ and all $(t, s) \in[(2 n+1) \delta, 2(n+1) \delta]$,

$$
\left\|u^{\delta}(t)-u^{\delta}(s)\right\|_{L^{1}(\mathbb{R})} \leq C_{0}|t-s| .
$$

Taking into account that $u^{\delta}(s, \cdot) \in W^{1,1}(\mathbb{R})$ and the estimates of $(6.1)$, some classical cuttings of integrals involving approximate units give, for all $\left.\delta \in] 0, \delta_{0}\right]$, all $t>0$, all $s \geq 0$ and all $\eta>0$,

$$
\left\|K(t) * u^{\delta}(s)-u^{\delta}(s)\right\|_{L^{1}(\mathbb{R})} \leq 2\left\|u_{0}\right\|_{L^{1}(\mathbb{R})} \int_{|y| \geq \eta} K(t, y) d y+\eta\left\|u_{0}^{\prime}\right\|_{L^{1}(\mathbb{R})}
$$


Let us now prove the equicontinuity of $\left.\left.\left\{u^{\delta}, \delta \in\right] 0, \delta_{0}\right]\right\}$ in $C\left(\left[0, \infty\left[; L^{1}(\mathbb{R})\right)\right.\right.$. Let $\left.\delta \in] 0, \delta_{0}\right]$ and $0 \leq t<s$. Let $p \leq q$ be integers such that $p \delta \leq t<(p+1) \delta$ and $q \delta \leq s<(q+1) \delta$; because of the different behaviours of $u^{\delta}$ (see $(6.2)$ ), we must separate the cases depending on the parity of $p$ and $q$; since all these cases are similar, we study only one, for example $p$ even and $q$ odd.

The idea, to estimate $\left\|u^{\delta}(s, \cdot)-u^{\delta}(t, \cdot)\right\|_{L^{1}(\mathbb{R})}$ is to go from $u^{\delta}(q \delta)$ to $u^{\delta}((p+1) \delta)$ by the following technique: on the intervals where $u^{\delta}$ satisfies the regularizing equation, we use the formula $u^{\delta}(k \delta)=K(2 \delta) * u^{\delta}((k-1) \delta)$ (hence for $k$ odd) and, on the intervals where $u^{\delta}$ satisfies the hyperbolic problem, we write $u^{\delta}(k \delta)=$ $u^{\delta}((k-1) \delta)+\left(u^{\delta}(k \delta)-u^{\delta}((k-1) \delta)\right)(k$ even $)$, the second term being estimated by $(6.3)$.

Applying this idea, using the semi-group property of the convolution by $K(t)$ and recalling that $q$ is odd in our example, an induction allows to see that, for all $l \in[0,(q-1) / 2]$,

$u^{\delta}(q \delta)=K(2 l \delta) * u^{\delta}((q-2 l) \delta)+\sum_{j=1}^{l} K(2 j \delta) *\left(u^{\delta}((q-2 j+1) \delta)-u^{\delta}((q-2 j) \delta)\right)$

(if $l=0, \sum_{j=1}^{l}(\ldots)$ is null and $K(2 l \delta) * u^{\delta}((q-2 l) \delta)$ is replaced by $u^{\delta}(q \delta)$ ). Taking $l=(q-p-1) / 2 \in[0,(q-1) / 2]$ (recall that $q$ is odd and $p$ is even and inferior to $q$, thus $p+1 \leq q)$ in this formula, we obtain

$$
\begin{aligned}
u^{\delta}(s) & =u^{\delta}(s)-u^{\delta}(q \delta)+K((q-p-1) \delta) * u^{\delta}((p+1) \delta) \\
& +\sum_{j=1}^{(q-p-1) / 2} K(2 j \delta) *\left(u^{\delta}((q-2 j+1) \delta)-u^{\delta}((q-2 j) \delta)\right) .
\end{aligned}
$$

Since $p$ is even, by definition of $u^{\delta}$ on $\left.] p \delta,(p+1) \delta\right]$ and $(2.5)$, we have $u^{\delta}((p+1) \delta)=$ $K(2((p+1) \delta-t)) *\left(K(2(t-p \delta)) * u^{\delta}(p \delta)\right)=K(2((p+1) \delta-t)) * u^{\delta}(t)$. We can therefore write

$$
\begin{aligned}
u^{\delta}(s)-u^{\delta}(t)= & u^{\delta}(s)-u^{\delta}(q \delta) \\
& +K((q-p-1) \delta+2((p+1) \delta-t)) * u^{\delta}(t)-u^{\delta}(t) \\
& +\sum_{j=1}^{(q-p-1) / 2} K(2 j \delta) *\left(u^{\delta}((q-2 j+1) \delta)-u^{\delta}((q-2 j) \delta)\right) .
\end{aligned}
$$

On $[q \delta, s] \subset[q \delta,(q+1) \delta]$ and each $[(q-2 j) \delta,(q-2 j+1) \delta]$ for $j \in[1,(q-p-1) / 2]$, $u^{\delta}$ satisfies the hyperbolic problem; thus, by (6.3) and (6.4), we have, for all $\eta>0$,

$$
\begin{aligned}
& \left\|u^{\delta}(s)-u^{\delta}(t)\right\|_{L^{1}(\mathbb{R})} \leq C_{0}|s-q \delta|+\frac{q-p-1}{2} C_{0} \delta \\
& \quad+2\left\|u_{0}\right\|_{L^{1}(\mathbb{R})} \int_{|y| \geq \eta} K((q-p-1) \delta+2((p+1) \delta-t), y) d y+\eta\left\|u_{0}^{\prime}\right\|_{L^{1}(\mathbb{R})} .
\end{aligned}
$$


But, since $t<(p+1) \delta \leq q \delta \leq s$, we have $(q-p-1) \delta=q \delta-(p+1) \delta \leq s-t$, $2((p+1) \delta-t) \leq 2(s-t)$ and $s-q \delta \leq s-t$. Using these bounds in the preceding inequality, we obtain, for all $\left.\delta \in] 0, \delta_{0}\right]$, for all $0 \leq t<s$ and for all $\eta>0$,

$$
\begin{aligned}
& \left\|u^{\delta}(s)-u^{\delta}(t)\right\|_{L^{1}(\mathbb{R})} \leq \frac{3 C_{0}}{2}|s-t| \\
& \quad+2\left\|u_{0}\right\|_{L^{1}(\mathbb{R})} \sup _{\tau \in] 0,3|s-t|]} \int_{|y| \geq \eta} K(\tau, y) d y+\eta\left\|u_{0}^{\prime}\right\|_{L^{1}(\mathbb{R})}
\end{aligned}
$$

(the same kind of formula can be obtained in the cases where $p$ and $q$ have other parities than the ones considered here).

Since, for all $\eta>0, \sup _{\tau \in] 0,3|s-t|]} \int_{|y| \geq \eta} K(\tau, y) d y \rightarrow 0$ as $|s-t| \rightarrow 0$ (property of an approximate unit), (6.5) gives the desired equicontinuity and concludes the proof of Proposition 6.1.

\subsection{Passing to the limit $\delta \rightarrow 0$}

By Proposition 6.1, we can suppose, up to a subsequence, that, for all $T>0$ and all $Q$ compact subset of $\mathbb{R}, u^{\delta} \rightarrow u$ in $C\left([0, T] ; L^{1}(Q)\right)$ as $\delta \rightarrow 0$. For all $t \geq 0, u^{\delta}(t) \rightarrow$ $u(t)$ in $L_{\text {loc }}^{1}(\mathbb{R})$, hence almost everywhere on $\mathbb{R}$ up to a subsequence. We deduce thus from (6.1) and Fatou's lemma that, for all $t>0,\|u(t)\|_{L^{1}(\mathbb{R})} \leq\left\|u_{0}\right\|_{L^{1}(\mathbb{R})}$, that

$$
\forall t \geq 0, \quad\|u(t)\|_{L^{\infty}(\mathbb{R})} \leq\left\|u_{0}\right\|_{L^{\infty}(\mathbb{R})}
$$

and that, as $u^{\delta}$, the function $u$ takes its values between the minimum and maximum values of $u_{0}$. Still using Fatou's lemma on subsequences (depending on $s$ and $t$ ), we see that (6.5) is satisfied for all $(s, t) \in\left[0, \infty\left[\right.\right.$ with $u$ instead of $u^{\delta}$; hence, $u \in C\left(\left[0, \infty\left[; L^{1}(\mathbb{R})\right)\right.\right.$ and, since $u^{\delta}(0)=u_{0}$ for all $\delta>0$, we have $u(0)=u_{0}$.

We now show that $u$ satisfies (1.1) if we use a formulation involving test functions.

Proposition 6.2 For all $\gamma \in C_{c}^{\infty}(] 0, \infty[)$ and all $\varphi \in \mathcal{S}(\mathbb{R})$, we have

$$
\int_{\mathbb{R}^{+} \times \mathbb{R}} u(t, x) \gamma^{\prime}(t) \varphi(x)+f(u(t, x)) \gamma(t) \varphi^{\prime}(x)-u(t, x) \gamma(t) g[\varphi](x) d x d t=0 .
$$

\section{Proof of Proposition 6.2}

Let $\left.\delta \in] 0, \delta_{0}\right]$. If $p$ is an odd integer, $u^{\delta}$ is a regular solution to $\partial_{t} u^{\delta}+$ $2 \partial_{x}\left(f\left(u^{\delta}\right)\right)=0$ on $[p \delta,(p+1) \delta] \times \mathbb{R}$. Multiplying this equation by $\gamma(t) \varphi(x)$ and integrating by parts (recall that $u^{\delta}$ is bounded and that $\varphi \in \mathcal{S}(\mathbb{R})$ ), we find

$$
\begin{aligned}
0= & -\int_{p \delta}^{(p+1) \delta} \int_{\mathbb{R}} u^{\delta}(t, x) \gamma^{\prime}(t) \varphi(x)+2 f\left(u^{\delta}(t, x)\right) \gamma(t) \varphi^{\prime}(x) d t d x \\
& +\int_{\mathbb{R}} u^{\delta}((p+1) \delta, x) \gamma((p+1) \delta) \varphi(x) d x-\int_{\mathbb{R}} u^{\delta}(p \delta, x) \gamma(p \delta) \varphi(x) d x .
\end{aligned}
$$


If $p$ is an even integer, then $u^{\delta}(t)=K(2(t-p \delta)) * u^{\delta}(p \delta)$ on $\left.] p \delta,(p+1) \delta\right] \times \mathbb{R}$. Since $u^{\delta}(t) \in L^{1}(\mathbb{R})$ for all $t \geq 0$, Fubini's theorem allows to write

$$
\mathcal{F}^{-1}\left(u^{\delta}(t)\right)=\mathcal{F}^{-1}(K(2(t-p \delta))) \mathcal{F}^{-1}\left(u^{\delta}(p \delta)\right)=e^{-2(t-p \delta)|\cdot|^{\lambda}} \mathcal{F}^{-1}\left(u^{\delta}(p \delta)\right) .
$$

Writing $\varphi=\mathcal{F}^{-1}(\mathcal{F}(\varphi))$ and $g[\varphi]=\mathcal{F}^{-1}\left(|\cdot|^{\lambda} \mathcal{F}(\varphi)\right)$, since $u^{\delta}(t) \in L^{1}(\mathbb{R})$ for all $t \geq 0$, by Fubini's theorem we can put the inverse Fourier transform on $u^{\delta}$ and we thus check that

$$
\begin{aligned}
& \int_{p \delta}^{(p+1) \delta} \int_{\mathbb{R}} u^{\delta}(t, x) \gamma^{\prime}(t) \varphi(x)-2 u^{\delta}(t, x) \gamma(t) g[\varphi](x) d x d t \\
& \quad=\int_{\mathbb{R}} u^{\delta}((p+1) \delta, x) \gamma((p+1) \delta) \varphi(x)-u^{\delta}(p \delta, x) \gamma(p \delta) \varphi(x) d x .
\end{aligned}
$$

Summing (6.8) on all odd integers $p$ and (6.9) on all even integers $p$ (notice that, since the support of $\gamma$ is compact, these sums are finite), the boundary terms disappear (even for $p=0$ since $\gamma(0)=0$ ) and we find

$$
\begin{aligned}
& \int_{\mathbb{R}^{+}} \int_{\mathbb{R}} u^{\delta}(t, x) \varphi(x) \gamma^{\prime}(t) d x d t \\
& +\int_{\mathbb{R}^{+}} \int_{\mathbb{R}^{2}} 2 f\left(u^{\delta}(t, x)\right) \varphi^{\prime}(x) \gamma(t)\left(1-\chi_{\delta}(t)\right) d x d t \\
& -\int_{\mathbb{R}^{+}} \int_{\mathbb{R}^{2}} 2 u^{\delta}(t, x) g[\varphi](x) \gamma(t) \chi_{\delta}(t) d x d t=0
\end{aligned}
$$

where $\chi_{\delta}$ is the characteristic function of $\left.\left.\cup_{\text {even }}\right] p \delta,(p+1) \delta\right]$.

Taking $T \geq \max (\operatorname{supp}(\gamma))$, we have, for all $A \geq 0$, thanks to (6.1) and (6.6)

$$
\begin{aligned}
& \left|\int_{\mathbb{R}^{+}} \int_{\mathbb{R}} u^{\delta}(t, x) g[\varphi](x) \gamma(t) 2 \chi_{\delta}(t) d x d t-\int_{\mathbb{R}^{+}} \int_{\mathbb{R}} u(t, x) g[\varphi](x) \gamma(t) d x d t\right| \\
& \leq\left|\int_{0}^{T} \int_{-A}^{A}\left(2 \chi_{\delta}(t) u^{\delta}(t, x)-u(t, x)\right) g[\varphi](x) \gamma(t) d x d t\right| \\
& \quad+3\left\|u_{0}\right\|_{L^{\infty}(\mathbb{R})} T\|\gamma\|_{L^{\infty}\left(\mathbb{R}^{+}\right)} \int_{\mathbb{R} \backslash[-A, A]}|g[\varphi](x)| d x
\end{aligned}
$$

Since $g[\varphi]$ is bounded on $\mathbb{R}, u^{\delta} \rightarrow u$ in $C\left([0, T] ; L^{1}([-A, A])\right)$ and $\chi_{\delta} \rightarrow 1 / 2$ in $L^{\infty}(] 0, \infty[)$ weak-* as $\delta \rightarrow 0$, we see that (6.11) tends to 0 as $\delta \rightarrow 0$. By Proposition 5.2 , we have $g[\varphi]=g_{1} * \varphi+g_{2} * \varphi^{(4)} \in L^{1}(\mathbb{R})$; hence, (6.12) tends to 0 as $A \rightarrow \infty$. We deduce thus that, as $\delta \rightarrow 0$,

$$
\int_{\mathbb{R}^{+}} \int_{\mathbb{R}} u^{\delta}(t, x) g[\varphi](x) \gamma(t) 2 \chi_{\delta}(t) d x d t \rightarrow \int_{\mathbb{R}^{+}} \int_{\mathbb{R}} u(t, x) g[\varphi](x) \gamma(t) d x d t .
$$


The flux function $f$ is lipschitz-continuous on $\left[-\left\|u_{0}\right\|_{L^{\infty}(\mathbb{R})},\left\|u_{0}\right\|_{L^{\infty}(\mathbb{R})}\right]$ so that, by (6.1) and (6.6), $f\left(u^{\delta}\right) \rightarrow f(u)$ in $C\left([0, T] ; L^{1}(Q)\right)$ for all $T>0$ and all compact subset $Q$ of $\mathbb{R}$. Therefore, with the same kind of reasoning as before, we can pass to the limit $\delta \rightarrow 0$ in (6.10) to conclude that $u$ satisfies (6.7).

We now prove that $u$ is in fact a solution to (1.1) in the sense of Definition 3.1.

Recall that $u \in C\left(\left[0, \infty\left[; L^{1}(\mathbb{R})\right)\right.\right.$. By (6.6) and the local lipschitz-continuity of $f$, we have $f(u) \in C\left(\left[0, \infty\left[; L^{1}(\mathbb{R})\right)\right.\right.$ (recall that $\left.f(0)=0\right)$. We deduce, since $\mathcal{F}^{-1}: L^{1}(\mathbb{R}) \rightarrow C_{b}(\mathbb{R})$ is continuous, that $t \rightarrow \mathcal{F}^{-1}(u(t))$ and $t \rightarrow \mathcal{F}^{-1}(f(u(t)))$ are in $C\left(\left[0, \infty\left[; C_{b}(\mathbb{R})\right) \subset C\left(\left[0, \infty[\times \mathbb{R})\right.\right.\right.\right.$. Hence, for all $\gamma \in \mathcal{C}_{c}^{\infty}(] 0, \infty[)$, the function

$w(\xi)=\int_{\mathbb{R}^{+}} \mathcal{F}^{-1}(u(t))(\xi) \gamma^{\prime}(t)+2 i \pi \xi \mathcal{F}^{-1}(f(u(t)))(\xi) \gamma(t)-\mathcal{F}^{-1}(u(t))(\xi)|\xi|^{\lambda} \gamma(t) d t$,

is continuous on $\mathbb{R}$. Let $\psi \in C_{c}^{\infty}(\mathbb{R})$; applying (6.7) with $\varphi=\mathcal{F}^{-1}(\psi) \in \mathcal{S}(\mathbb{R})$ and using Fubini's theorem, we have $\int_{\mathbb{R}} w \psi=0$; the function $\psi$ being arbitrary, this implies $w \equiv 0$. Since this is true for all $\gamma \in C_{c}^{\infty}(] 0, \infty[)$, we deduce that, for all $\xi \in \mathbb{R}, \frac{d}{d t}\left(\mathcal{F}^{-1}(u(\cdot))(\xi)\right)=-|\xi|^{\lambda} \mathcal{F}^{-1}(u(\cdot))(\xi)+2 i \pi \xi \mathcal{F}^{-1}(f(u(\cdot)))(\xi)$ in $\mathcal{D}^{\prime}(] 0, \infty[)$. The right-hand side of this equation is a continuous function, and the equation is therefore a classical ODE; thus, for all $\xi \in \mathbb{R}$ and all $t \geq 0$,

$$
\begin{aligned}
\mathcal{F}^{-1}(u(t))(\xi) & =e^{-t|\xi|^{\lambda}} \mathcal{F}^{-1}\left(u_{0}\right)(\xi)+\int_{0}^{t} 2 i \pi \xi e^{-(t-s)|\xi|^{\lambda}} \mathcal{F}^{-1}(f(u(s)))(\xi) d s \\
& =\mathcal{F}^{-1}\left(K(t) * u_{0}\right)(\xi)-\int_{0}^{t} \mathcal{F}^{-1}\left(\partial_{x} K(t-s)\right) \mathcal{F}^{-1}(f(u(s)))(\xi) d s \\
& =\mathcal{F}^{-1}\left(K(t) * u_{0}\right)(\xi)-\int_{0}^{t} \mathcal{F}^{-1}\left(\partial_{x} K(t-s) * f(u(s))\right)(\xi) d s
\end{aligned}
$$

By (2.4) and since $f(u) \in C\left(\left[0, \infty\left[; L^{1}(\mathbb{R})\right)\right.\right.$, Fubini's theorem gives then

$$
\mathcal{F}^{-1}(u(t))(\xi)=\mathcal{F}^{-1}\left(K(t) * u_{0}\right)(\xi)-\mathcal{F}^{-1}\left(\int_{0}^{t} \partial_{x} K(t-s) * f(u(s)) d s\right)(\xi) .
$$

$\mathcal{F}^{-1}$ being injective on $L^{1}(\mathbb{R})$, we deduce that $u$ satisfies $(3.1)$ on $] 0, \infty[\times \mathbb{R}$.

Here is a summary of what we have proved so far in this section.

Proposition 6.3 If $u_{0} \in C_{c}^{\infty}(\mathbb{R})$, then there exists a solution to (1.1) on $] 0, \infty[$ which is bounded by $\left\|u_{0}\right\|_{L^{\infty}(\mathbb{R})}$ and takes its values between the minimum and maximum values of $u_{0}$.

\subsection{Conclusion}

We now prove that if $u_{0} \in L^{\infty}(\mathbb{R})$, then there exists a solution to (1.1) on ]0, $\infty$ [ which satisfies item ii) in Theorem 1.1, which concludes the proof of this theorem. 
Let $u_{0} \in L^{\infty}(\mathbb{R})$ and take $\left(u_{0}^{n}\right)_{n \geq 0} \in C_{c}^{\infty}(\mathbb{R})$ which converges a.e. on $\mathbb{R}$ to $u_{0}$ and such that, for all $n \geq 1, u_{0}^{n}$ takes its values between the essential lower and upper bounds of $u_{0}$; in particular, $\left\|u_{0}^{n}\right\|_{L^{\infty}(\mathbb{R})} \leq\left\|u_{0}\right\|_{L^{\infty}(\mathbb{R})}$ for all $n \geq 0$. Denote by $u^{n}$ a solution, given by Proposition 6.3 , to (1.1) on ]0, $\infty$ [ with initial condition $u_{0}^{n}$ instead of $u_{0} ; u^{n}$ is bounded by $\left\|u_{0}^{n}\right\|_{L^{\infty}(\mathbb{R})} \leq\left\|u_{0}\right\|_{L^{\infty}(\mathbb{R})}$. This bound and Theorem 5.1 show that, for all $t_{0}>0$ and all $m \geq 0,\left(\partial_{x}^{m} u^{n}\right)_{n \geq 1}$ is bounded on $] t_{0}, \infty[\times \mathbb{R}$; by Lemma 5.1 and Proposition 5.2, these bounds on the spatial derivatives imply that $\left(\partial_{t} u^{n}\right)_{n \geq 1}$ is also bounded on $] t_{0}, \infty[\times \mathbb{R}$.

Hence, by Ascoli-Arzela's theorem, up to a subsequence, we can suppose that there exists $u$ such that $u^{n} \rightarrow u$ on $] 0, \infty\left[\times \mathbb{R}\right.$. Since $\left\|u^{n}\right\|_{L^{\infty}(] 0, \infty[\times \mathbb{R})} \leq\left\|u_{0}\right\|_{L^{\infty}(\mathbb{R})}$, we also have $\|u\|_{L^{\infty}(] 0, \infty[\times \mathbb{R})} \leq\left\|u_{0}\right\|_{L^{\infty}(\mathbb{R})}$ and, in fact, $u$ takes (as each $u^{n}$ ) its values between the essential lower and upper bounds of $u_{0}$. The function $u^{n}$ satisfies (3.1) with $u_{0}^{n}$ instead of $u_{0}$; passing to the limit $n \rightarrow \infty$ in this equation, thanks to the dominated convergence theorem, we see that $u$ is a solution to (1.1) on $] 0, \infty[$.

Remark 6.2 Since both the hyperbolic and regularizing equations satisfy the properties given in Remark 1.2, it is quite obvious, on our construction of a solution to (1.1), that (1.1) also satisfies the properties stated in Remark 1.2 (because we can always choose approximations of the initial conditions by regular data which satisfy the hypotheses of this remark).

\section{References}

[BCP68] J-M. Bony, P. Courrège, and P. Priouret, Semi-groupes de Feller sur une variété à bord compacte et problèmes aux limites intégro-différentiels du second ordre donnant lieu au principe du maximum, Ann. Inst. Fourier (Grenoble) 18 (1968), no. fasc. 2, 369-521 (1969).

[CD01] P. Clavin and B. Denet, Theory of cellular detonations in gases. part 2. mach-stem formation at strong overdrive, C. R. Acad. Sci. Paris t. 329 (2001), no. Série II b, 489-496.

[CD02] _ Diamond patterns in the cellular front of an overdriven detonation, Phys. Rev. Letters 88 (2002).

[CH01] P. Clavin and L. He, Theory of cellular detonations in gases. part 1. stability limits at strong overdrive, C. R. Acad. Sci. Paris t. 329 (2001), no. Série II b, 463-471.

[FJS01] W. Farkas, N. Jacob, and R. L. Schilling, Feller semigroups, $L^{p}{ }_{-s u b-}$ Markovian semigroups, and applications to pseudo-differential operators with negative definite symbols, Forum Math. 13 (2001), no. 1, 51-90. 
[Hoh95] W. Hoh, Pseudodifferential operators with negative definite symbols and the martingale problem, Stochastics Stochastics Rep. 55 (1995), no. 3-4, $225-252$.

[Kru70] S. N. Kruzhkov, First order quasilinear equations with several independent variables., Mat. Sb. (N.S.) 81 (123) (1970), 228-255.

[Lév25] P. Lévy, Calcul des Probabilités, 1925.

[Vol67] A. I. Vol'pert, Spaces bv and quasilinear equations, Mat. Sb. (N.S.) 73 (115) (1967), 255-302. 